\title{
A posteriori error analysis for elliptic PDEs on domains with complicated structures
}

\author{
Carstensen, C ; Sauter, Stefan A
}

\begin{abstract}
The discretisation of boundary value problems on complicated domains cannot resolve all geometric details such as small holes or pores. The model problem of this paper consists of a triangulated polygonal domain with holes of a size of the mesh-width at most and mixed boundary conditions for the Poisson equation. Reliable and efficient a posteriori error estimates are presented for a fully numerical discretisation with conforming piecewise affine finite elements. Emphasis is on technical difficulties with the numerical approximation of the domain and their influence on the constants in the reliability and efficiency estimates.
\end{abstract}

DOI: https://doi.org/10.1007/s00211-003-0495-4

Posted at the Zurich Open Repository and Archive, University of Zurich ZORA URL: https://doi.org/10.5167/uzh-21775

Journal Article

Originally published at:

Carstensen, C; Sauter, Stefan A (2004). A posteriori error analysis for elliptic PDEs on domains with complicated structures. Numerische Mathematik, 96(4):691-721.

DOI: https://doi.org/10.1007/s00211-003-0495-4 


\title{
A POSTERIORI ERROR ANALYSIS FOR ELLIPTIC PDES ON DOMAINS WITH COMPLICATED STRUCTURES
}

\author{
CARSTEN CARSTENSEN AND STEFAN A. SAUTER
}

\begin{abstract}
The discretisation of boundary value problems on complicated domains cannot resolve all geometric details such as small holes or pores. The model problem of this paper consists of a triangulated polygonal domain with holes of a size of the mesh-width at most and mixed boundary conditions for the Poisson equation. Reliable and efficient a posteriori error estimates are presented for a fully numerical discretisation with conforming piecewise affine finite elements. Emphasis is on technical difficulties with the numerical approximation of the domain and their influence on the constants in the reliability and efficiency estimates.
\end{abstract}

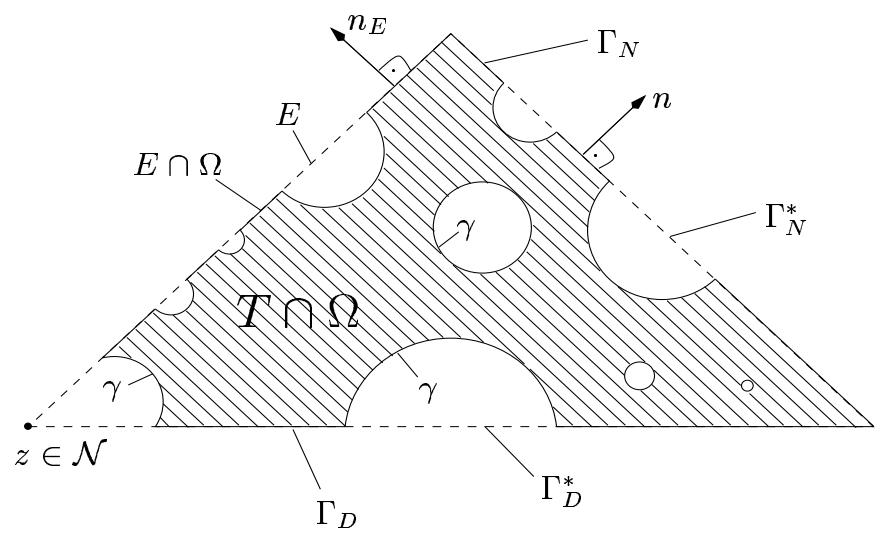

FIGURE 1. Intersection of a triangle $T$ with the domain $\Omega$ at the boundary.

Date: June 7, 2001.

1991 Mathematics Subject Classification. 65N30, 65R20, 73C50.

Key words and phrases. finite element method, a posteriori error estimates, adaptive algorithm, reliability, efficiency. 


\section{INTRODUCTION}

Porous media or advanced materials with microstructures provide examples for boundary value problems with small geometric details. Typically, those details cannot be completely resolved by the mesh of a finite element discretisation, but have to be involved. This work is devoted to the mathematical analysis for the Poisson equation on a domain with holes of a mesoscale: Large holes are resolved by the finite element mesh exactly, but holes of the diameter of the mesh-size and smaller are not, as illustrated in Fig. 1. Efficient and reliable a posteriori error estimates are derived for a conforming piecewise affine finite element scheme on a triangulation which covers a bigger domain $\Omega^{\star}$ that includes the domain $\Omega$ with holes inside and on the surface.

For elliptic problems on complicated domains, the minimal dimension of any finite element space is huge since the finite element mesh has to resolve the geometry. Thus, from the viewpoint of accuracy and balancing of local errors, we cannot expect that the degrees of freedom of such a finite element space are distributed in a (nearly) optimal way. In [HS1], composite finite element spaces have been introduced where the minimal number of unknowns is independent of the size and number of geometric details. The combination of composite finite element spaces with an a posteriori error estimator (used as an error indicator) allows to design problem-adapted finite element spaces where the adaptation process starts from very coarse levels.

In addition, by using this a posteriori error estimator the finite element error can be estimated on discretisation levels where not all geometric details are resolved by the mesh (but taking them into account by using composite finite element functions).

Our paper is devoted to the definition and analysis of a reliable and efficient a posteriori error estimator. As a model problem we will study the Poisson problem $-\Delta u=f$ with mixed boundary conditions. We will consider a Lipschitz domain $\Omega$ which arises by removing from a polygonal domain $\Omega^{\star}$ a possibly huge number of holes. "Holes" are simple connected domains which are collected in the set of holes $\mathcal{C}$. We suppose homogeneous Neumann boundary conditions on the boundary of holes, e.g., for small bubbles of gas.

The discretisation is based on a conforming triangulation $\mathcal{T}$ of the overlapping domain $\Omega^{\star}$ with continuous, piecewise affine finite elements and their restrictions to the domain $\Omega$. In a first phase, a $\mathcal{T}$-piecewise affine discrete function $U^{\star}$ is computed on $\Omega^{\star}$ while the approximation of the continuous solution $u$ is given by $U:=\left.U^{\star}\right|_{\Omega}$. 


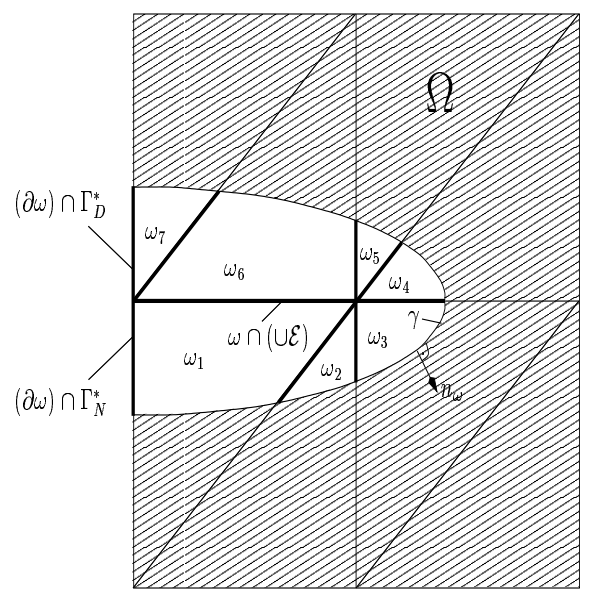

FiguRE 2. Intersection of a hole $\omega$ with $\cup \mathcal{E}$.

Our reliable a posteriori error estimator will be presented in Section 3. Besides error residuals, we obtain, for an interior hole $\omega \in \mathcal{C}$, the term

$$
\eta_{\omega}^{2}:=h_{\omega} \int_{\partial \omega}\left|\partial U / \partial n_{\omega}\right|^{2} d s
$$

corresponding to $\partial u / \partial n_{\omega}=0$ on $\partial \omega$ (and modifications for any hole which touches the boundary).

We carefully study the efficiency of this contribution where difficulties arise from the fact that $\omega$ may be intersected with edges of the triangulation $\mathcal{T}$ in a quite arbitrary and complicated way; compare Figure 2 for an illustration.

Our main result can be stated as follows. Suppose $u \in H^{1}(\Omega)$ denotes the exact solution and $U=\left.U^{\star}\right|_{\Omega}$ its discrete approximation. If all integrals are evaluated exactly (otherwise we obtain inconsistency error sources $\left.\eta_{c}\right)$, the error in energy norm $\|\nabla(u-U)\|_{L^{2}(\Omega)}$ is bounded by

$$
\eta:=\left\|h_{\mathcal{T}} f\right\|_{L^{2}(\Omega)}+\left\|h_{\mathcal{E}}^{1 / 2}\left[\partial U^{\star} / \partial n_{\mathcal{E}}\right]\right\|_{L^{2}(\cup \mathcal{E})}+\left(\sum_{\omega \in \mathcal{C}} \eta_{\omega}^{2}\right)^{1 / 2}
$$

where $h_{\mathcal{T}}$ (resp. $h_{\mathcal{E}}, h_{\omega}$ ) is the local mesh-size (resp. edge-size, holesize), and $\left[\partial U^{\star} / \partial n_{\mathcal{E}}\right]$ is the jump of the normal components of two $(\mathcal{T}$-piecewise) discrete gradients across the edges (and standard modifications on the boundary). Theorem 3.1 implies (for exactly matched Dirichlet boundary conditions) the reliability of $\eta$ in the sense of

$$
\|\nabla(u-U)\|_{L^{2}(\Omega)} \leq c_{1} \eta .
$$


Theorem 6.1 shows efficiency, i.e., the converse inequality

$$
c_{2} \eta \leq\|\nabla(u-U)\|_{L^{2}(\Omega)}+\text { h.o.t. }
$$

In the latter inequality, h.o.t. are known higher order terms and it holds in a local form. The constants $c_{1}$ and $c_{2}$ are independent of mesh-sizes or hole-sizes. They depend on some features of the geometry of holes. For instance, $c_{1}$ stays uniformly bounded if the holes are circular with diameter $h_{\omega} \leq c_{3} h_{T}$ for neighbouring elements $T$ of size $h_{T}$ provided a separation condition is satisfied, namely, two distinct holes $\omega_{1}$ and $\omega_{2}$ have a distance $\operatorname{dist}\left(\omega_{1}, \omega_{2}\right)$ with $h_{\omega_{1}}+h_{\omega_{2}} \leq c_{4} \operatorname{dist}\left(\omega_{1}, \omega_{2}\right)$. To bound $c_{2}$ from below, we will assume in addition that $h_{\omega}<c_{4} \operatorname{dist}\left(\omega, \Gamma^{\star}\right)$ holds. It is stressed that, then, $c_{1}$ and $c_{2}$ are independent of the way the edges intersect with holes and tiny pieces as well as entire edges may lie inside the hole.

As a setting for this, the behaviour of the constants appearing in some trace estimates and in estimates of norms of appropriate extension operators on some geometry parameters will be investigated in Section 4 . The reliability of the a posteriori error estimator (stated in Theorem 3.1) will be proved in Section 5. The conditions sufficient for the efficiency estimate of Theorem 6.1 may appear technical at first glance. Therefore, included examples illustrate the consequences of Assumptions 6.1 till 6.8. The proof in Section 7, however, clearly underlines that the assumptions posed are natural. The analysis of edge and volume contributions per se requires minor modifications of standard techniques $[\mathrm{V}]$ while the investigations of the hole contributions are more involved. We emphasise that, in contrast to [DR], where the effect of approximating the boundary of the domain is incorporated in the error estimator, our finite element spaces are defined on the true domain while the construction allows a low-dimensional discretisation even for very complicated domains.

\section{Model PROBLEM}

As a model problem we consider a domain $\Omega \subset \mathbb{R}^{2}$ which arises by removing holes from a polygonal domain. More precisely, let $\Omega^{\star} \subset \mathbb{R}^{2}$ denote a polygonal domain with boundary $\Gamma^{\star}=\partial \Omega^{\star}$ and let $\mathcal{C}=$ $\left\{\omega_{j}: 1 \leq j \leq J\right\}$ be a countable set of simply connected Lipschitz domains $\omega_{j}$, the 'holes', which have a positive distance from each other and are all (not necessarily compactly) contained in $\Omega^{\star}$. The physical domain $\Omega:=\Omega^{\star} \backslash \overline{\bigcup \mathcal{C}}$ is supposed to be Lipschitz (as a further assumption on the intersections of the hole boundaries $\partial \omega$ with $\left.\Gamma^{\star}\right)$. Mixed boundary conditions are imposed on the boundary $\Gamma:=\partial \Omega$, namely 
homogeneous Neumann boundary conditions on $\gamma:=\Omega^{\star} \cap \partial(\cup \mathcal{C})$, prescribed Neumann data $g$ on $\Gamma_{N}:=\left(\Gamma \cap \Gamma^{\star}\right) \backslash \Gamma_{D}$, and prescribed Dirichlet data $u_{D}$ on $\Gamma_{D} \subset \Gamma \cap \Gamma^{\star}$ of positive length.

Remark 2.1. Note that $\Gamma_{D} \cup \Gamma_{N}=\Gamma^{\star}$ if $\Omega^{\star}$ includes all holes compactly. Otherwise, the inclusion $\Gamma_{D} \cup \Gamma_{N} \subset \Gamma^{\star}$ can be strict.

The strong formulation of the continuous problem reads: Given $f \in$ $L^{2}(\Omega), g \in L^{2}\left(\Gamma_{N}\right)$, and $u_{D} \in H^{1}\left(\Gamma_{D}\right)$, seek $u \in H^{1}(\Omega)$ satisfying

$$
\begin{array}{cl}
\Delta u+f=0 \quad \text { in } \Omega, & u=u_{D} \quad \text { on } \Gamma_{D}, \\
\partial u / \partial n=g \quad \text { on } \Gamma_{N}, & \partial u / \partial n=0 \quad \text { on } \gamma .
\end{array}
$$

Since the normal derivative of $u$ vanishes at interior boundaries of holes, the weak formulation reads: Seek $u \in H^{1}(\Omega)$ with $\left.u\right|_{\Gamma_{D}}=u_{D}$ and

$$
\int_{\Omega} \nabla u \cdot \nabla v d x=\int_{\Omega} f v d x+\int_{\Gamma_{N}} g v d s
$$

for all test functions $v \in H_{D}^{1}(\Omega):=\left\{v \in H^{1}(\Omega):\left.v\right|_{\Gamma_{D}}=0\right\}$.

The discretisation of the model problem is based on composite finite elements which are defined in three steps (i)-(iii) [HS1].

(i) The (overlapping) triangulation. The polygonal domain $\Omega^{\star}$ is partitioned exactly by a regular triangulation $\mathcal{T}$ into closed triangles $T \in \mathcal{T}$ in the sense of Ciarlet $[\mathrm{BS}, \mathrm{Ci}], \overline{\Omega^{\star}}=\cup \mathcal{T}$. Two non-disjoint distinct triangles in $\mathcal{T}$ share either a common edge $E$ or a vertex $z$ called node. The set of all edges resp. nodes is denoted by $\mathcal{E}$ resp. $\mathcal{N}$. Edges on the boundary $\Gamma^{\star}$ (belong to only one triangle and) are collected in the set $\mathcal{E}_{\Gamma^{\star}}=\mathcal{E}_{D} \cup \mathcal{E}_{N}$, where $\mathcal{E}_{\Gamma^{\star}}$ is split into edges of Dirichlet- and Neumann type as follows. Let $\left|E \cap \Gamma_{D}\right|$ and $\left|E \cap \Gamma_{N}\right|$ denote the onedimensional measure of the sets $E \cap \Gamma_{D}$ and $E \cap \Gamma_{N}$, respectively, along the edge $E \in \mathcal{E}_{\Gamma^{\star}}$; suppose that either $\left|E \cap \Gamma_{D}\right|$ or $\left|E \cap \Gamma_{N}\right|$ is positive (but not both of them). Then, set $\mathcal{E}_{D}:=\left\{E \in \mathcal{E}_{\Gamma^{\star}}:\left|E \cap \Gamma_{D}\right|>0\right\}$ and $\mathcal{E}_{N}:=\mathcal{E}_{\Gamma^{\star}} \backslash \mathcal{E}_{D}, \mathcal{E}_{\Omega}:=\mathcal{E} \backslash \mathcal{E}_{\Gamma^{\star}}$, and $\Gamma_{N}^{\star}:=\cup \mathcal{E}_{N}$ resp. $\Gamma_{D}^{\star}:=\cup \mathcal{E}_{D}$.

(ii) The (overlapping) finite element space. Let $\mathcal{S}^{\star}$ denote the space of $\mathcal{T}$-piecewise affine finite elements and $\mathcal{S}_{D}^{\star}$ its subspace with vanishing traces on $\Gamma_{D}^{\star}$, i.e.,

$$
\begin{aligned}
& \mathcal{S}^{\star}:=\left\{V \in C\left(\Omega^{\star}\right): \forall T \in \mathcal{T},\left.V\right|_{T} \text { is affine on } T\right\}, \\
& \mathcal{S}_{D}^{\star}:=\left\{V \in \mathcal{S}^{\star}:\left.V\right|_{\Gamma_{D}^{\star}}=0\right\} .
\end{aligned}
$$


(iii) The (restricted) composite finite element space. The spaces $\mathcal{S}$ and $\mathcal{S}_{D}$ are given by

$$
\begin{aligned}
\mathcal{S} & :=\left.\mathcal{S}^{\star}\right|_{\Omega}:=\left\{V \in C(\Omega): \exists V^{\star} \in \mathcal{S}^{\star}, V=\left.V^{\star}\right|_{\Omega}\right\}, \\
\mathcal{S}_{D} & :=\left.\mathcal{S}_{D}^{\star}\right|_{\Omega} .
\end{aligned}
$$

Remark 2.2. Using $\Gamma_{D} \subset \Gamma^{\star}$ we have

$$
\mathcal{S}_{D}^{\star}=\left\{V \in \mathcal{S}^{\star}: \forall E \in \mathcal{E}_{D},\left.V\right|_{E}=0\right\} .
$$

Remark 2.3. Throughout the paper we write $u, v$, for functions lying in the infinite dimensional space $H^{1}(\Omega), H_{D}^{1}(\Omega)$ and $U, V, \ldots$ for functions in the finite element space $\mathcal{S}, \mathcal{S}_{D}$. Functions on the extended domain $\Omega^{\star}$ have a superscript $\star$, e.g., $u^{\star}, U^{\star}$. Approximations to the right-hand sides and the porosity (see below) are denoted with a tilde superscript as, e.g., $\tilde{f}, \tilde{g}$, while $f^{\star}$ is the extension of $f$ from $\Omega$ to $\Omega^{\star}$ by zero. If $v$ and $v^{\star}$ appear in the same context, we understand $v=\left.v^{\star}\right|_{\Omega}$.

The intersection of an element with the domain and some notations are illustrated in Fig. 1. Note carefully that domains are open and connected and that $T \in \mathcal{T}$ and $E \in \mathcal{E}$ are closed sets in $\mathbb{R}^{2}$; the interior of $T$ is denoted by $\operatorname{int}(T)$.

To define a fully numerical discretisation, we need to approximate Dirichlet data $u_{D} \in H^{1}\left(\Gamma_{D}\right)$ by the trace of some function $U_{D}^{\star} \in \mathcal{S}^{\star}$. The finite element scheme requires a stiffness matrix where, for each element $T \in \mathcal{T}$ of area $|T|$, some constant $\left.\tilde{\varrho}\right|_{T}$ approximates the ratio $\varrho_{T}:=|\Omega \cap T| /|T|$, where $\tilde{\varrho} \in \mathcal{L}^{0}(\mathcal{T})$ and $\mathcal{L}^{0}(\mathcal{T})$ denotes the $\mathcal{T}$-piecewise constants. Furthermore, the computation involves approximate righthand sides $\tilde{f}^{\star} \in L^{2}\left(\Omega^{\star}\right)$ and $\tilde{g}^{\star} \in L^{2}\left(\Gamma_{N}^{\star}\right)$. Then, the discrete problem reads: Seek $U^{\star} \in \mathcal{S}^{\star}$ and $U:=\left.U^{\star}\right|_{\Omega}$ satisfying $U^{\star}=U_{D}^{\star}$ on $\Gamma_{D}^{\star}$ and, for all $V^{\star} \in \mathcal{S}_{D}^{\star}$,

$$
\int_{\Omega^{\star}} \tilde{\varrho} \nabla U^{\star} \cdot \nabla V^{\star} d x=\int_{\Omega^{\star}} \tilde{f}^{\star} V^{\star} d x+\int_{\Gamma_{N}^{\star}} \tilde{g}^{\star} V^{\star} d s .
$$

Remark 2.4. Notice that the computational cost for solving the discrete problem (2.4) are small as the geometry is not resolved in detail (use of $\Omega^{\star}, \Gamma_{D}^{\star}, \Gamma_{N}^{\star}$ instead of $\left.\Omega, \Gamma_{D}, \Gamma_{N}\right)$ and holes are taken into account only via an approximate porosity. In the error analysis, those errors shall be taken into account.

Remark 2.5. The integrals over intersections $T \cap \Omega^{\star}$ should be evaluated by using a composite quadrature rule employing a hierarchical resolution of the domain (cf., e.g., [HS2, OR]). The efficient implementation of the a posteriori controlled discretisation scheme presented here will 
be the topic of a forthcoming paper. Some ideas on the adaptive numerical integration will be sketched at the end of Section 3.

\section{A POSTERIORI ERROR ESTIMATE}

Suppose $u \in H^{1}(\Omega)$ is the exact solution of $(2.2)$ and let $U=\left.U^{\star}\right|_{\Omega}$ be the restriction of the discrete solution of (2.4). The ingredients of the error estimator for the energy error of $u-U$ are the volume residual $\eta_{\Omega}$, the edge contributions $\eta_{E}$, the hole errors $\eta_{\mathcal{C}}$, the Dirichlet contribution $\eta_{D}$, and the consistency term $\eta_{c}$.

Let $h_{\mathcal{T}}\left(\right.$ resp. $\left.h_{\mathcal{E}}\right)$ be a $\mathcal{T}$-piecewise (resp. $\mathcal{E}$-piecewise) constant weight to measure the mesh-size (resp. edge-size) regarded as $L^{\infty}$-functions on $\Omega^{\star}$ (resp. on $\left.\cup \mathcal{E}\right)$. Then, the volume contribution reads

$$
\eta_{\Omega}:=\left\|h_{\mathcal{T}} f\right\|_{L^{2}(\Omega)} .
$$

For each edge $E \in \mathcal{E}$, let $n_{E}$ denote one unit normal on $E$ with fixed orientation (oriented to the exterior if $\left.E \in \mathcal{E}_{\Gamma^{*}}\right)$ and $n_{\mathcal{E}} \in L^{\infty}(\cup \mathcal{E})$ denotes their composition, i.e., $\left.n_{\mathcal{E}}\right|_{E}:=n_{E}$. The $\mathcal{T}$-piecewise constant discrete gradient $\nabla U$ has a jump across each inner edge $E \in \mathcal{E}_{\Omega}$ and the difference $\left.(\nabla U)\right|_{T_{+}}-\left.(\nabla U)\right|_{T_{-}}$is denoted as $[\nabla U]$ on $E ; T_{+}$and $T_{-}$are the two distinct elements which share the edge $E$ such that $n_{E}$ points into $T_{+}$. Note that the jump of the normal components $[\nabla U] \cdot n_{E}=:\left.\left[\partial U / \partial n_{\mathcal{E}}\right]\right|_{E}$ is independent of the chosen orientation of $n_{E}$. We regard $n_{\mathcal{E}}$ and $\left[\partial U / \partial n_{\mathcal{E}}\right]$ as $\mathcal{E}$-piecewise constant functions on the skeleton $\cup \mathcal{E}$ of edges; $\cup \mathcal{E}$ is the set of all points $x$ on some boundary $\partial T$ of some triangle $T \in \mathcal{T}$. Then, the edge contribution reads

$$
\eta_{\mathcal{E}}:=\left\|h_{\mathcal{E}}^{1 / 2}\left[\frac{\partial U^{\star}}{\partial n_{\mathcal{E}}}\right]\right\|_{L^{2}\left(\cup \mathcal{E}_{\Omega}\right)}+\left\|h_{\mathcal{E}}^{1 / 2}\left(g^{\star}-\frac{\partial U^{\star}}{\partial n}\right)\right\|_{L^{2}\left(\Gamma_{N}^{\star}\right)} .
$$

Owing to homogeneous Neumann conditions on $\gamma$, the analogue to the edge contribution defines the hole contributions,

$$
\eta_{\mathcal{C}}:=\left(\sum_{\omega \in \mathcal{C}} h_{\omega} \int_{\partial \omega \backslash \Gamma_{D}^{\star}}\left|\partial U^{\star} / \partial n_{\omega}\right|^{2} d s\right)^{1 / 2}
$$

( $h_{\omega}$ denotes the diameter of $\omega \in \mathcal{C}$ ). In case that $u_{D}$ is the restriction of a smooth function on $\Gamma_{D}^{\star}$, the Dirichlet contribution

$$
\eta_{D}:=\min \left\{\|\nabla v\|_{L^{2}(\Omega)}: v \in H^{1}(\Omega) \text { such that } v=u_{D}-U \text { on } \Gamma_{D}\right\}
$$


is of higher order (and then may be neglected). Finally, the abstract consistency term is given by

$$
\begin{aligned}
\eta_{c}:= & \sup _{\substack{W^{\star} \in \mathcal{S}_{D}^{\star} \\
\left\|W^{\star}\right\|_{H^{1}\left(\Omega^{\star}\right)}=1}}\left\{\int_{\Omega^{\star}}\left(f^{\star}-\tilde{f}^{\star}\right) W^{\star} d x\right. \\
& \left.+\int_{\Gamma_{N}^{\star}}\left(g^{\star}-\tilde{g}^{\star}\right) W^{\star} d s-\int_{\Omega^{\star}}(\varrho-\tilde{\varrho}) \nabla U^{\star} \cdot \nabla W^{\star} d x\right\} .
\end{aligned}
$$

Theorem 3.1. There exists an $\left(h_{\mathcal{T}}, h_{\mathcal{E}}, u, U, f, g\right)$-independent positive constant $c_{1}$ such that

$$
\|\nabla(u-U)\|_{L^{2}(\Omega)} \leq c_{1}\left(\eta_{\Omega}+\eta_{E}+\eta_{\mathcal{C}}+\eta_{D}+\eta_{c}\right) .
$$

The constant $c_{1}$ depends on the domain $\Omega, \Gamma_{D}, \Gamma_{N}$, and the shape of the elements in $\mathcal{T}$ (such as their aspect ratio) but neither on the number or size of the holes in $\mathcal{C}$ nor the way they are hit by $\cup \mathcal{E}$.

Sections 4 and 5 are devoted to the proof of the theorem while efficiency will be studied in Sections 6 and 7 .

A few remarks and examples on the evaluation of the error estimator will conclude this section.

The evaluation of the term $\eta_{\mathcal{E}}$ can be performed as described in $[\mathrm{V}]$ since $U^{\star}$ is a standard finite element function and $\mathcal{E}_{\Omega}$ resp. $\Gamma_{N}^{\star}$ consists of triangle edges.

The remaining terms in the error estimator concern integrals over the intersections $T \cap \Omega$ resp. $T \cap \partial \omega$ which can be realized by adaptively subdividing $T$; a triangle $T$ is subdivided regularly by connecting the midpoints of edges.

The triangulation $\mathcal{T}(T)$, which is generated for numerical integration purposes only, is the result of " $\mathcal{T}(T):=\emptyset ; \operatorname{refine}(T, \mathcal{T}(T))$ ".

procedure refine $(T, \mathcal{T})$;

\section{begin}

if $T \cap \Omega$ is a simple domain, i.e., the integrals

$$
\int_{T \cap \Omega} \ldots d x, \quad \int_{T \cap \partial \omega} \ldots d s, \quad \int_{T \cap \Gamma_{N}} \ldots d s
$$

can be approximated by standard quadrature formulae,

then $\mathcal{T}:=\mathcal{T} \cup\{T\}$ 


\section{else begin}

subdivide $T$ regularly to obtain the children $\left\{T_{j}\right\}_{j=1}^{4}$;

for all $1 \leq j \leq 4$ with $\operatorname{int}\left(T_{j}\right) \cap \Omega \neq \emptyset$ do refine $\left(T_{j}, \mathcal{T}\right) ;$

\section{end; end;}

By using the subdivisions $\mathcal{T}(T)$, the contribution $\eta_{\mathcal{C}}$ can be evaluated by

$$
\eta_{\mathcal{C}}^{2}=\sum_{\omega \in \mathcal{C}} h_{\omega} \sum_{\substack{T \in \mathcal{T} \\ T \cap \partial \omega \neq \emptyset}} \sum_{K \in \mathcal{T}(T)} \int_{K \cap\left(\partial \omega \backslash \Gamma_{D}^{\star}\right)} \chi\left(n \cdot \nabla q_{T}\right)^{2} d s
$$

where $q_{T}:=\nabla\left(\left.U^{\star}\right|_{T}\right)$ and $\chi: \partial \omega \rightarrow\{1 / 2,1\}$ is given by

$$
\chi(x):= \begin{cases}1 & \text { if there is only one } K \in \mathcal{T}(T) \text { with } x \in K, \\ 1 / 2 & \text { otherwise. }\end{cases}
$$

Since the main focus of this paper is the investigation of the hole contributions to the finite element error we discuss the error terms corresponding to the data approximation $\eta_{D}$ and $\eta_{c}$ under simplifying assumptions.

Example 3.1. Suppose that there is a function $U_{D}^{\star} \in \mathcal{S}^{\star}$ with $u_{D}=$ $\left.U_{D}^{\star}\right|_{\Gamma_{D}}$. Then, the Dirichlet contribution vanishes by making the ansatz $U^{\star}=U_{D}^{\star}+U_{0}^{\star}$ and solving for $U_{0}^{\star}$. Note that $U_{0}^{\star}$ vanishes at the Dirichlet boundary.

Example 3.2. If there exists a continuous $u_{D}^{\star}: \Gamma_{D}^{\star} \rightarrow \mathbb{R}$ with $u_{D}=$ $\left.u_{D}^{\star}\right|_{\Gamma_{D}}$ which is $\mathcal{E}_{D}$-piecewise smooth, i.e., $\left.u_{D}^{\star}\right|_{E} \in H^{2}(E)$ for all $E \in \mathcal{E}_{D}$, $\eta_{D}$ can be of higher order. Indeed, it is proved in Lemma 4.1 of [CB] that, if $U^{\star}(z)=u_{D}^{\star}(z)$ for all nodes $z \in \mathcal{N} \cap \Gamma_{D}$, then

$$
\eta_{D} \leq c_{5}\left\|h_{\mathcal{E}}^{3 / 2} \partial^{2} u_{D}^{\star} / \partial s^{2}\right\|_{L^{2}\left(\Gamma_{D}^{\star}\right)},
$$

where $\partial / \partial s$ denotes the derivative along $E$.

Example 3.3. Assume that the data $f, g$ are sufficiently smooth. We may extend the function $f \in L^{2}(\Omega)$ to $f^{\star} \in L^{2}\left(\Omega^{\star}\right)$ and $g \in L^{2}\left(\Gamma_{N}\right)$ to $g^{\star} \in L^{2}\left(\Gamma_{N}^{\star}\right)$ by zero. In order to evaluate the integrals, we employ the subdivision $\mathcal{T}(T)$ to obtain

$$
\int_{\Omega^{\star}} f^{\star} V^{\star} d x=\int_{\Omega} f V d x=\sum_{T \in \mathcal{T}} \int_{\Omega \cap T} f V d x=\sum_{T \in \mathcal{T}} \sum_{K \in \mathcal{T}(T)} \int_{\Omega \cap K} f V d x .
$$

Since we assumed that the integrals over $\Omega \cap K$ can be approximated sufficiently accurate and $f$ is smooth, the corresponding consistency term in $\eta_{c}$ can be neglected. In a similar fashion, the integral over 
$\Gamma_{N}^{\star}$ reduces to an integral over $\Gamma_{N}$ and the subdivisions $\mathcal{T}(T)$ can be employed for numerical integration as in (3.6).

Remark 3.1. By an adaptive resolution of the boundary generated by the procedure refine, the amount of work for realising our finite element method is proportional to the number of elements in $\mathcal{T}$ plus the number of subdivided elements for resolving the curved boundary and, in this way, depends linearly on the number of geometric details.

\section{Extension operators, trace theorems, and Poincaré INEQUALITIES ON COMPLICATED DOMAINS}

The proof of the reliability and the efficiency of the error estimator is based on estimates of the norm of certain extension and trace operators. In this section, we will show that their norms are moderately bounded for a broad class of domains which might contain a huge number of small holes.

4.1. Extension operators. Let $\Omega \subset \Omega^{\star}, \Gamma_{D}, \Gamma_{D}^{\star}$, etc. be as in Section 2 and $H_{D}^{1}\left(\Omega^{\star}\right):=\left\{v \in H^{1}\left(\Omega^{\star}\right):\left.v\right|_{\Gamma_{D}^{\star}}=0\right\}$. In this section, we will define an extension operator $\mathfrak{E}_{D}: H_{D}^{1}(\Omega) \rightarrow H_{D}^{1}\left(\Omega^{\star}\right)$ so that the supremum

$$
\sup _{v \in H_{D}^{1}(\Omega) \backslash\{0\}}\left\|\nabla \mathfrak{E}_{D} v\right\|_{L^{2}\left(\Omega^{\star}\right)} /\|\nabla v\|_{L^{2}(\Omega)}=: c_{6}<\infty .
$$

is moderately bounded for large class of domains, which may contain a huge number of geometric details.

Remark 4.1. The constant $c_{1}$ will depend on $c_{6}$.

The extension operator $\mathfrak{E}_{D}$ is constructed in three steps. Let $u \in$ $H_{D}^{1}(\Omega)$. Since $\Omega$ is a Lipschitz domain it is well known that there exists an extension operator $\mathfrak{E}^{\text {Stein }}: H_{D}^{1}(\Omega) \rightarrow H^{1}\left(\Omega^{\star}\right)$. Put $u_{1}^{\star}:=$ $\mathfrak{E}^{\mathrm{Stein}} u \in H^{1}\left(\Omega^{\star}\right)$ and note that $\left.u\right|_{\Gamma_{D}}=0$. Next, we employ a function $m: \Omega^{\star} \rightarrow[0,1]$ satisfying $m \equiv 1$ on $\Omega$ and $m=0$ on $\Gamma_{D}^{\star} \backslash \Gamma_{D}$ to define $u_{2}^{\star}:=m u_{1}^{\star} \in H_{D}^{1}\left(\Omega^{\star}\right)$. Finally, the local Ritz-projections $u_{2}^{0}$ of $u_{2}^{\star}$ on $\omega \in \mathcal{C}$ is subtracted to end up with $u^{\star}:=u_{2}^{\star}-u_{2}^{0}=: \mathfrak{E}_{D} u$. The details of this construction along with illustrating examples will be discussed in this section.

Theorem 4.1 (Stein). Let $\Omega \subset \mathbb{R}^{d}$ be a bounded Lipschitz domain. Then, there exists an extension operator $\mathfrak{E}^{\text {Stein }}: H_{D}^{1}(\Omega) \rightarrow H^{1}\left(\Omega^{\star}\right)$. 


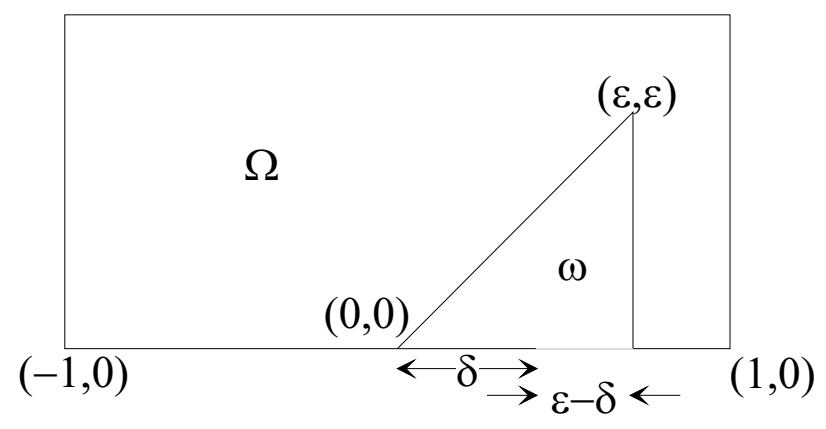

FiguRE 3. Domain $\Omega$ with triangular hole $\omega$ and a change of the type of boundary conditions outside $\partial \Omega$ to illustrate Assumption 4.1.

For a proof, we refer to $[\mathrm{S}]$. Theorem 4.1 neither implies that the operator norm of $\mathfrak{E}^{\text {Stein }}$ is moderately bounded (the bound might be very large for domains with a huge number of small geometric details) nor that the $H^{1}$-seminorm of the extended function can be estimated by the $H^{1}$-seminorm of the original function. To fulfil homogeneous Dirichlet boundary conditions on $\Gamma_{D}^{\star}$, we assume the existence of an appropriate cutoff-function.

Assumption 4.1. There exists a function $m: \overline{\Omega^{\star}} \rightarrow[0,1]$ such that $m \equiv 1$ on $\Omega$ while $m=0$ on $\Gamma_{D}^{\star} \backslash \Gamma_{D}$ and, for all $v \in H^{1}\left(\Omega^{\star}\right)$ with $v=0$ on $\Gamma_{D}$, we have that the product $m v$ belongs to $H_{D}^{1}\left(\Omega^{\star}\right)$. Set

$$
\mathfrak{M}:\left\{v \in H^{1}\left(\Omega^{\star}\right):\left.v\right|_{\Gamma_{D}}=0\right\} \rightarrow H_{D}^{1}\left(\Omega^{\star}\right), \quad v \mapsto m v
$$

For holes $\omega \in \mathcal{C}$, which do not touch the exterior Dirichlet boundary $\Gamma_{D}^{\star} \backslash \Gamma_{D}$, we may choose $\left.m\right|_{\omega} \equiv 1$. The following example considers the characteristic model situation of Figure 3 .

Example 4.1. Let $\Omega=\Omega^{\star} \backslash \bar{\omega}$ where $\Omega^{\star}=(-1,1) \times(0,1)$ and

$$
\omega:=\operatorname{int} \operatorname{conv}\{(0,0),(\varepsilon, 0),(\varepsilon, \varepsilon)\} \quad \text { for some } 0<\varepsilon<1 / 2 \text {. }
$$

(Recall that int and conv denote the interior and convex hull, respectively, of a set.) Suppose $\Gamma_{D}^{\star}:=[-1, \delta] \times\{0\}$ for some $0<\delta<\varepsilon$, while $\Gamma_{N}^{\star}:=\Gamma^{\star} \backslash \overline{\Gamma_{D}^{\star}}, \Gamma_{D}=[-1,0] \times\{0\}$. Let $(x, y)=r(\cos \alpha, \sin \alpha)$. We define the function $m$ by

$$
m(x, y):= \begin{cases}1 & \text { if } x \in \bar{\Omega} \\ \chi(x)+(1-\chi(x)) \sin (2 \alpha) & \text { if } x \in \omega\end{cases}
$$


where $\chi(x)=0$ for $0<x<\delta$ and $\chi(x)=(x-\delta) /(\varepsilon-\delta)$ for $\delta \leq x \leq \varepsilon$. It is easy to check that $m$ is continuous in the open set $\Omega^{\star}$ and $m=0$ on $\Gamma_{D}^{\star} \backslash \Gamma_{D}$. Given $v_{1}^{\star} \in H^{1}\left(\Omega^{\star}\right)$ with $\left.v_{1}^{\star}\right|_{\Gamma_{D}}=0$, we define $v_{2}^{\star}:=m v_{1}^{\star}$. The proof of

$$
\|m\|_{L^{\infty}\left(\Omega^{\star}\right)}=1 \quad \text { and } \quad|\nabla m(x)| \leq \frac{2+\sqrt{2}}{r(1-\delta / \varepsilon)} \quad \text { for all } x \in \Omega^{\star}
$$

is straightforward. Hence, Hardy's inequality in the form of Theorem 1.4.44 in [G] (where $s=1, p=2, \alpha=0$ ) yields $\mathfrak{M} v_{1}^{\star} \in H^{1}\left(\Omega^{\star}\right)$ and so eventually $v_{2}^{\star} \in H_{D}^{1}\left(\Omega^{\star}\right)$.

In the next step, we introduce the Ritz-projection of functions $H_{D}^{1}\left(\Omega^{\star}\right)$ in the space $\mathcal{V}:=\left\{v \in H^{1}\left(\Omega^{\star}\right):\left.v\right|_{\Omega \cup \Gamma_{D}^{\star}}=0\right\}$.

Definition 4.1. The Ritz-projection $\mathfrak{R}: H_{D}^{1}\left(\Omega^{\star}\right) \rightarrow \mathcal{V}$ is given for $v_{1}^{\star} \in H_{D}^{1}\left(\Omega^{\star}\right)$ by $v_{2}^{\star}:=\mathfrak{R} v_{1}^{\star}$ where $v_{2}^{\star} \in \mathcal{V}$ is the solution of

$$
\int_{\Omega^{\star}} \nabla v_{2}^{\star} \cdot \nabla w=\int_{\Omega^{\star}} \nabla v_{1}^{\star} \cdot \nabla w d x \quad \text { for all } w \in \mathcal{V} .
$$

Now, we have all ingredients for defining the extension operator $\mathfrak{E}_{D}$.

Definition 4.2. The extension operator $\mathfrak{E}_{D}: H_{D}^{1}(\Omega) \rightarrow H_{D}^{1}\left(\Omega^{\star}\right)$ is given by the composition

$$
\mathfrak{E}_{D}:=(I-\mathfrak{R}) \mathfrak{M E}^{\mathrm{Stein}}
$$

Following the ideas in [OSY], it was proved in [SW] that the norm of the extension operator $\mathfrak{E}_{D}$ does not depend on the size and number of holes in the domain provided a certain separation condition (see Section 1, (4.2), and [SW, (2.8)]) is satisfied.

To reduce technicalities we focus on some characteristic examples and refer to $[\mathrm{SW}]$ for proofs and general considerations.

Example 4.2. Let $\Omega^{\star} \subset \mathbb{R}^{d}$ denote a Lipschitz domain and $\left\{B_{j}\right\}_{j \in N}$, $N \subseteq \mathbb{N}$, be a family of balls with radius $\varepsilon_{j}$ which are compactly included in $\Omega^{\star}$ and satisfy a separation condition

$$
\operatorname{dist}\left(B_{j}, B_{k}\right) \geq c_{7} \max \left\{\varepsilon_{j}, \varepsilon_{k}\right\} \text { and } \operatorname{dist}\left(B_{j}, \Gamma^{\star}\right) \geq c_{7} \varepsilon_{j}
$$

for all distinct $j, k \in N$ and the global constant $c_{7}>0$. Let $\Omega:=$ $\Omega^{\star} \backslash \overline{\bigcup B_{j \in N}}$. Choose $m \equiv 1$ in $\Omega^{\star}$ (cf. Assumption 4.1) and define $\mathfrak{E}_{D}$ as in (4.1). Then, the operator norm of $\mathfrak{E}_{D}$ and its seminorm, i.e., the constant $c_{6}$, is bounded independently of $\operatorname{card}(N)$ or $\varepsilon_{j}$. 
Example 4.3. For $\delta>0$, let $\Omega^{\star}=(-1-\delta, 1+\delta)^{2}$ and $\omega=(-1,1)^{2}$. Then, there exists a constant $c_{8}>0$ so that the norm of every extension operator $\mathfrak{E}: H^{1}\left(\Omega^{\star} \backslash \bar{\omega}\right) \rightarrow H^{1}\left(\Omega^{\star}\right)$ can be estimated from below by

$$
c_{8} \delta^{-1 / 2} \leq\|\mathfrak{E}\| .
$$

The following example shows that the separation condition (4.2) is not necessary in order to bound the norm of the minimal extension operator by a moderate constant.

Example 4.4. Let $\Omega^{\star}=(-1,1)^{3}$ and, for $j=1,2, \omega_{j}=B_{j} \times(-1,1)$. Here, $B_{j}$ denotes the disc with radius $\varepsilon$ about the points $( \pm 2 \varepsilon, 0)^{\top}$. Then, the norm of the minimal extension operator $\mathfrak{E}: H^{1}\left(\Omega^{\star} \backslash \overline{\omega_{1} \cup \omega_{2}}\right)$ $\rightarrow H^{1}\left(\Omega^{\star}\right)$ is bounded uniformly as $\varepsilon \rightarrow 0$.

Finally, we revisit Example 4.1 and estimate the norm of the extension operator.

Example 4.5. Let $\Omega, \Omega^{\star}, \omega$, and the function $m$ be defined as in Example 4.1. Then, the norm and the seminorm of the extension operator $\mathfrak{E}_{D}$ as in (4.1) can be estimated form above by $C /(1-\delta / \varepsilon)$.

This example indicates that the (semi-)norm of the extension operator $\mathfrak{E}_{D}$ behaves critically if the ratio of the length of the Dirichlet portion $\partial \omega \cap \Gamma_{D}^{\star}$ compared to the length of the outer boundary $\partial \omega \cap \Gamma^{\star}$ tends to one.

4.2. Clément interpolation on complicated domains. The proof of the reliability of the error estimator makes use of the Clément approximation $[\mathrm{Cl}, \mathrm{V}, \mathrm{CF}]$ operator $P: H_{D}^{1}\left(\Omega^{\star}\right) \rightarrow \mathcal{S}_{D}^{\star}$ such that, for all $T \in \mathcal{T}$ and $u^{\star} \in H^{1}\left(\Omega^{\star}\right)$,

$$
\begin{aligned}
\left\|u^{\star}-P u^{\star}\right\|_{L^{2}(T)}+h_{T}\left|u^{\star}-P u^{\star}\right|_{H^{1}(T)} & \leq c_{9} h_{T}\left|u^{\star}\right|_{H^{1}\left(\omega_{T}\right)}, \\
\left\|h_{\mathcal{T}}^{-1}\left(u^{\star}-P u^{\star}\right)\right\|_{L^{2}\left(\Omega^{\star}\right)}+\left|u^{\star}-P u^{\star}\right|_{H^{1}\left(\Omega^{\star}\right)} & \leq c_{10}\left|u^{\star}\right|_{H^{1}\left(\Omega^{\star}\right)} .
\end{aligned}
$$

Here, $\omega_{T}:=\cup\{K \in \mathcal{T}: T \cap K \neq \emptyset\}$. The constants $c_{9}$ and $c_{10}$ depend merely on the aspect ratio of the elements. Their quantitative estimation is given in $[\mathrm{CF}]$. With $u^{\star}=\mathfrak{E}_{D} u$ we obtain that the righthand side in (4.4) can be bounded from above by $c_{11}|u|_{H^{1}(\Omega)}$ where $c_{11}$ depends on $c_{6}$ and $c_{10}$.

4.3. Trace theorems. Traces of $H^{1}$-functions along edges have to be estimated by their norms on adjacent triangles $[\mathrm{Cl}, \mathrm{CF}]$. For shape regular meshes, we have the local estimate for $u \in H^{1}(T)$ on the edge $E \subset \partial T, E \in \mathcal{E}, T \in \mathcal{T}$,

$$
\|u\|_{L^{2}(E)}^{2} \leq c_{12}\left(h_{E}^{-1}\|u\|_{L^{2}(T)}^{2}+h_{E}|u|_{H^{1}(T)}^{2}\right)
$$


and a global version, for $u^{\star} \in H^{1}\left(\Omega^{\star}\right)$,

$$
\left\|u^{\star}\right\|_{L^{2}(\cup \mathcal{E})} \leq c_{13}\left(\left\|h_{\mathcal{T}}^{-1 / 2} u^{\star}\right\|_{L^{2}\left(\Omega^{\star}\right)}+\left\|h_{\mathcal{T}}^{1 / 2} \nabla u^{\star}\right\|_{L^{2}\left(\Omega^{\star}\right)}\right) .
$$

Non-resolved geometric details require further estimates.

Definition 4.3. Let $\omega \subset \mathbb{R}^{2}$ denote a Lipschitz domain of area $|\omega|$ and let $\gamma \subset \bar{\omega}$ be a Lipschitz curve of length $|\gamma|$. The trace constant $C(\gamma, \omega)$ is

$$
C(\gamma, \omega):=\sup _{v \in H^{1}(\omega) \backslash\{0\}} \frac{\|v\|_{L^{2}(\gamma)}^{2}}{|\gamma| /|\omega|\|v\|_{L^{2}(\omega)}^{2}+|\omega| /|\gamma||v|_{H^{1}(\omega)}^{2}} .
$$

Remark 4.2. Letting $v \equiv 1$ in (4.5) shows $1 \leq C(\gamma, \omega)$.

The trace constant $C(\gamma, \omega)$ is scaling-invariant.

Lemma 4.1. For $\omega$ and $\gamma$ as in Definition 4.3 and $\varepsilon>0$, define $\chi_{\varepsilon}: \omega \rightarrow \omega_{\varepsilon}$ by $\chi_{\varepsilon}(x)=\varepsilon x$ and $\omega_{\varepsilon}=\chi_{\varepsilon}(\omega), \gamma_{\varepsilon}=\chi_{\varepsilon}(\gamma)$. Then, $C\left(\gamma_{\varepsilon}, \omega_{\varepsilon}\right)=C(\gamma, \omega)$.

Proof. Straightforward calculations yield $|v|_{H^{k}(\omega)}=\varepsilon^{k-1}\left|v \circ \chi_{\varepsilon}^{-1}\right|_{H^{k}\left(\omega_{\varepsilon}\right)}$, $k=0,1$, and $\|v\|_{L^{2}(\gamma)}^{2}=\varepsilon^{-1}\left\|v \circ \chi_{\varepsilon}^{-1}\right\|_{L^{2}\left(\gamma_{\varepsilon}\right)}^{2}$ for each $v \in H^{1}(\omega)$ and $\left|\omega_{\varepsilon}\right|=\varepsilon^{2}|\omega|$ or $\left|\gamma_{\varepsilon}\right|=\varepsilon|\gamma|$ from which we deduce the assertion.

Example 4.6. (a) Let $\omega$ be a disc with boundary $\gamma=\partial \omega$. Then, $C(\gamma, \omega) \leq 3$. (b) Let $\omega$ be a parallelogram and $\gamma$ one of its sides. Then, $C(\gamma, \omega) \leq 2$.

Proof. For the proof of (a) we refer to [BS, Sec. 1.6] and indicate the proof of (b) for rectangles (the case of a parallelogram is similar). Suppose $\omega=(0, a) \times(0, b)$. The mean value theorem guarantees that $f(\eta)=b^{-1} \int_{0}^{b} f(y) d y$ for $f \in H^{1}(0, b)$ and some $\eta \in(0, b)$. The fundamental theorem of calculus and Cauchy inequalities then show

$$
\begin{aligned}
& \quad f(0)^{2}=\left(b^{-1} \int_{0}^{b} f(y) d y-\int_{0}^{\eta} f^{\prime}(y) d y\right)^{2} \\
& \leq 2 b^{-2}\|f\|_{L^{1}(0, b)}^{2}+2\left\|f^{\prime}\right\|_{L^{1}(0, b)}^{2} \leq 2 b^{-1}\|f\|_{L^{2}(0, b)}^{2}+2 b\left\|f^{\prime}\right\|_{L^{2}(0, b)}^{2} .
\end{aligned}
$$

Replacing $f(y)$ in $(4.6)$ by $v(x, y)$ (and prime by $\partial / \partial y$ ) and integrating with respect to $x$ over $(0, a)$ we deduce $(b)$.

In the sequel, we will frequently estimate functions on (subsets of) holes and appropriate neighbourhoods thereof. 
Notation 4.1. For a set $A \subset \mathbb{R}^{2}$, the Chebyshev ball $B_{A}$ is the minimal ball that contains $A$. The disc with radius 2 diam $A$ about the midpoint of $B_{A}$ is denoted by $V_{A}$.

Lemma 4.2. Let $\omega \subset \mathbb{R}^{2}$ be a domain with diameter $h_{\omega}$ and let $\mathcal{T}$ be a shape regular triangulation of $\mathbb{R}^{2}$. Then,

$$
\|v\|_{L^{2}(\omega \cap(\cup \mathcal{E}))}^{2} \leq 2 c_{14}\left(h_{\omega}^{-1}\|v\|_{L^{2}\left(V_{\omega}\right)}^{2}+h_{\omega}|v|_{H^{1}\left(V_{\omega}\right)}^{2}\right) .
$$

The constant $c_{14}$ is the number of $\omega$-intersections with edges.

Proof. Let

$$
\mathcal{E}_{\omega}:=\{\omega \cap E: E \in \mathcal{E} \wedge|\omega \cap E|>0\} .
$$

For each $S \in \mathcal{E}_{\omega}$ define a rectangle $Q(S)$ with one side $S$ and the other of length $h_{\omega}$. Example 4.6(b) shows

$$
\frac{1}{2}\|v\|_{L^{2}(S)}^{2} \leq h_{\omega}^{-1}\|v\|_{L^{2}(Q(S))}^{2}+h_{\omega}|v|_{H^{1}(Q(S))}^{2}
$$

By definition, $c_{14}$ is the number of overlaps of $Q(S) \subset V_{\omega}$ for all $S \in \mathcal{E}_{\omega}$. This leads to

$$
\begin{aligned}
\frac{1}{2}\|v\|_{L^{2}\left(\cup \mathcal{E}_{\omega}\right)}^{2} \leq h_{\omega}^{-1} \sum_{S \in \mathcal{E}_{\omega}} \| & v \|_{L^{2}(Q(S))}^{2}+h_{\omega} \sum_{S \in \mathcal{E}_{\omega}}|v|_{H^{1}(Q(S))}^{2} \\
& \leq c_{14} h_{\omega}^{-1}\|v\|_{L^{2}\left(V_{\omega}\right)}^{2}+c_{14} h_{\omega}|v|_{H^{1}\left(V_{\omega}\right)}^{2}
\end{aligned}
$$

4.4. Poincaré inequalities. The proof of reliability and efficiency of the error estimators requires Poincaré inequalities [N, PW].

Theorem 4.2 (Payne and Weinberger). Let $\omega$ denote a convex domain in $\mathbb{R}^{2}$ with diameter $h_{\omega}$. Then, for all $u \in H^{1}(\omega)$ and $u_{\omega}:=$ $\int_{\omega} u d x /|\omega|$

$$
\left\|u-u_{\omega}\right\|_{L^{2}(\omega)} \leq h_{\omega} / \pi|u|_{H^{1}(\omega)} .
$$

For a nonconvex domain $\omega$, we first extend $u$ to a convex neighbourhood $V_{\omega}$ and then deduce

$$
\begin{aligned}
\left\|u-u_{\omega}\right\|_{L^{2}(\omega)} & \leq\left\|u-u_{V_{\omega}}\right\|_{L^{2}(\omega)} \leq\left\|u-u_{V_{\omega}}\right\|_{L^{2}\left(V_{\omega}\right)} \\
& \leq \operatorname{diam}\left(V_{\omega}\right) / \pi|u|_{H^{1}\left(V_{\omega}\right)} \leq c_{15} h_{\omega}|u|_{H^{1}(\omega)} .
\end{aligned}
$$




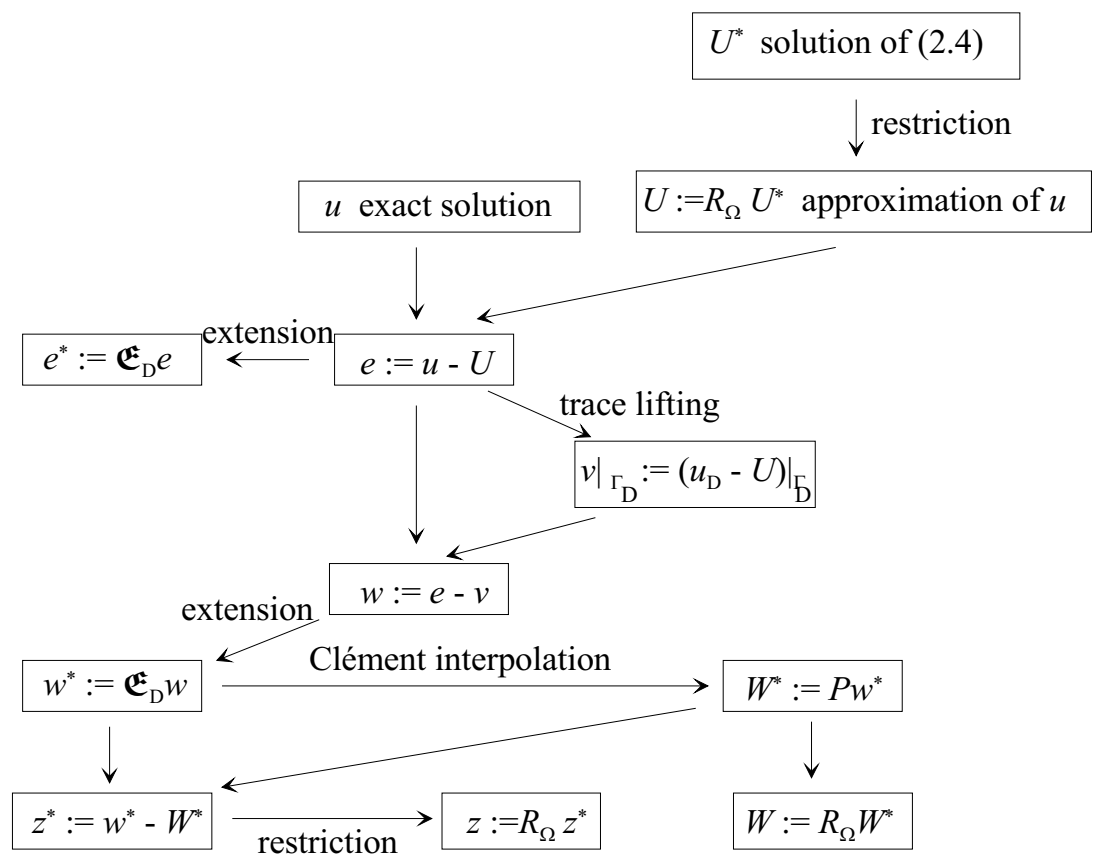

FIGURE 4. Diagram illustrating the relationship of the functions $U, U^{\star}, u, e, e^{\star}, v, w, w^{\star}, W^{\star}, W, z^{\star}$, and $z$.

\section{Proof of Reliability}

Throughout the proof we write $a \lesssim b$ for $a \leq c b$, where the multiplicative constant $c>0$ is independent of $h_{\mathcal{T}}, h_{\mathcal{E}}, u, U, f, g$ and may depend on $\Omega, \Gamma_{D}, \Gamma_{N}$, and on the shape of the elements or their aspect ratio. Furthermore, the estimates depend on the numbers

(5.1) $\max _{\omega \in \mathcal{C}} \operatorname{card}\{T \in \mathcal{T}: T \cap \omega \neq \emptyset\}$ and $\sup _{x \in \Omega^{\star}} \operatorname{card}\left\{\omega \in \mathcal{C}: x \in V_{\omega}\right\}$.

Since emphasis is on many small holes $\omega$ with $h_{\omega} \lesssim h_{T}$ (others shall be resolved in $\Omega^{\star}$ ) the numbers in (5.1) are moderate.

In the sequel, various functions arise with relationships illustrated in Figure 4. Recall that $U^{\star} \in \mathcal{S}^{\star}$ solves (2.4) and $U:=R_{\Omega} U^{\star}$, where $R_{\Omega}$ is the restriction of a function $v: \Omega^{\star} \rightarrow \mathbb{R}$ to $\Omega$. Split the error $e:=u-U \in H^{1}(\Omega)$ into $e-v$ and $v$, where $v \in H^{1}(\Omega)$ satisfies $v=u_{D}-U$ on $\Gamma_{D}$ and $\eta_{D}=\|\nabla v\|_{L^{2}(\Omega)}$. Given $w:=e-v \in H_{D}^{1}(\Omega)$ let $w^{\star}:=\mathfrak{E}_{D} w \in H_{D}^{1}\left(\Omega^{\star}\right)$ and let $W^{\star}:=P w^{\star}$ denote the Clément approximation to $w^{\star}$. Define $z^{\star}:=w^{\star}-W^{\star} \in H^{1}\left(\Omega^{\star}\right)$ and $z:=R_{\Omega} z^{\star}$, $W:=R_{\Omega} W^{\star}$. Observe $z \in H_{D}^{1}(\Omega)$ and $z^{\star}=0$ on $\Gamma_{D}^{\star}$. The $H^{1}$-norm of $W^{\star}$ can be estimated by using a Friedrichs inequality for $w^{\star} \in H_{D}^{1}\left(\Omega^{\star}\right)$, 
the approximation properties of the Clément approximation (4.4) and the continuity of the extension operator $\mathfrak{E}_{D}$ with respect to the $H^{1}$ seminorm

$$
\begin{aligned}
\left\|W^{\star}\right\|_{H^{1}\left(\Omega^{\star}\right)} & \leq\left\|W^{\star}-w^{\star}\right\|_{H^{1}\left(\Omega^{\star}\right)}+\left\|w^{\star}\right\|_{H^{1}\left(\Omega^{\star}\right)} \\
& \lesssim\left|w^{\star}\right|_{H^{1}\left(\Omega^{\star}\right)} \lesssim|e-v|_{H^{1}(\Omega)} .
\end{aligned}
$$

This, a triangle inequality with $\left|e^{\star}\right|_{H^{1}\left(\Omega^{\star}\right)} \lesssim|e|_{H^{1}(\Omega)}$, and $\left|U^{\star}-v^{\star}\right|_{H^{1}\left(\Omega^{\star}\right)} \lesssim$ $|e|_{H^{1}(\Omega)}$ at the end yield

$$
\begin{aligned}
\left\|h_{\mathcal{T}}^{-1} z^{\star}\right\|_{L^{2}\left(\Omega^{\star}\right)}+\left\|h_{\mathcal{E}}^{-1 / 2} z^{\star}\right\|_{L^{2}(\cup \mathcal{E})}+\left|z^{\star}\right|_{H^{1}\left(\Omega^{\star}\right)}+\left\|W^{\star}\right\|_{H^{1}\left(\Omega^{\star}\right)} \\
\lesssim|e-v|_{H^{1}(\Omega)} \lesssim|e|_{H^{1}(\Omega)}+\eta_{D} .
\end{aligned}
$$

The definition of $v$ implies $\int_{\Omega} \nabla v \cdot \nabla \varphi d x=0$ for all $\varphi \in H_{D}^{1}(\Omega)$. The choice $\varphi=e-v$ leads to

$$
\int_{\Omega} \nabla v \cdot \nabla e d x=\eta_{D}^{2}
$$

Hence, we obtain with $e=z+W+v$

$$
|e|_{H^{1}(\Omega)}^{2}=\int_{\Omega} \nabla e \cdot \nabla z d x+\int_{\Omega} \nabla e \cdot \nabla W d x+\eta_{D}^{2} .
$$

The second term on the right-hand side in (5.3) is split into $\nabla u \cdot \nabla W$ and $\nabla U \cdot \nabla W$. The concept of the porosity $\varrho$, the weak formulation (2.2), and (5.2) show (recall that $f^{\star}$ and $g^{\star}$ vanish outside $\Omega$ and $\Gamma_{N}$ )

$$
\begin{gathered}
\int_{\Omega} \nabla e \cdot \nabla W d x=\int_{\Omega^{\star}}\left(f^{\star}-\tilde{f}^{\star}\right) W^{\star} d x+\int_{\Gamma_{N}^{\star}}\left(g^{\star}-\tilde{g}^{\star}\right) W^{\star} d s \\
-\int_{\Omega^{\star}}(\varrho-\tilde{\varrho}) \nabla U^{\star} \cdot \nabla W^{\star} d x \leq\left\|W^{\star}\right\|_{H^{1}\left(\Omega^{\star}\right)} \eta_{c} \lesssim|e|_{H^{1}(\Omega)} \eta_{c} .
\end{gathered}
$$

For the first term on the right-hand side in (5.3), an integration by parts on each $T \cap \Omega$ is performed. Careful account on the exact boundary conditions results in

$$
\begin{aligned}
\int_{\Omega} \nabla e \cdot \nabla & z d x=\int_{\cup \mathcal{E} \cap \Omega}\left[\partial U / \partial n_{\mathcal{E}}\right] z d s \\
& +\int_{\Gamma_{N}}(g-\partial U / \partial n) z d s+\int_{\Omega} f z d x-\int_{\gamma} z \partial U / \partial n d s
\end{aligned}
$$

with jump terms on $\cup \mathcal{E} \cap \Omega:=(\cup \mathcal{E}) \cap \Omega$ within $\Omega$. Next, we will derive an appropriate representation of the last integral in (5.5). Figure 2 illustrates how edges and boundary pieces might hit a hole $\omega \in \mathcal{C}$.

Let us consider one hole $\omega \in \mathcal{C}$ with outer normal $n_{\omega}=-n_{\Omega}=-n$ and boundary $\partial \omega=\gamma_{\omega} \cup \gamma_{D}^{\star} \cup \gamma_{N}^{\star}$, where $\gamma_{\omega}:=(\partial \omega) \cap \gamma, \gamma_{D}^{\star}:=(\partial \omega) \cap \Gamma_{D}^{\star}$, and 
$\gamma_{N}^{\star}:=(\partial \omega) \cap \Gamma_{N}^{\star}$. The edges cut $\omega$ into a finite number of connectivity components $\omega_{1}, \ldots, \omega_{J}$ illustrated in Fig. 2, $\omega \backslash(\cup \mathcal{E})=\cup\left\{\omega_{1}, \ldots, \omega_{J}\right\}$. (Their number $J \lesssim 1$ is limited since $\omega$ intersects with only a finite number of elements, cf. (5.1)). On each $\omega_{j}, \nabla U^{\star}$ is constant and equal to $\left.\nabla U^{\star}\right|_{\omega_{j}}$. The divergence theorem shows

$$
\int_{\partial \omega_{j}} \partial U^{\star} / \partial n_{\omega_{j}} d s=\left.\nabla U^{\star}\right|_{\omega_{j}} \cdot \int_{\partial \omega_{j}} n_{\omega_{j}} d s=0 .
$$

Therefore, for any real constant $c_{\omega}$, we obtain

$$
\int_{\partial \omega_{j}} z^{\star} \partial U^{\star} / \partial n_{\omega_{j}} d s=\int_{\partial \omega_{j}}\left(z^{\star}-c_{\omega}\right) \partial U^{\star} / \partial n_{\omega_{j}} d s .
$$

Note that $\nabla U^{\star}$ is, in general, discontinuous across $\cup \mathcal{E}_{\Omega}$. Besides the situation in Figure 2 it may happen that $\gamma_{\omega}$ has a positive intersection with the skeleton $\cup \mathcal{E}_{\Omega},\left|\gamma_{\omega} \cap\left(\cup \mathcal{E}_{\Omega}\right)\right|>0$. Even in this case, we have

$$
\partial U / \partial n=-\partial U^{\star} / \partial n_{\omega}-\left[\partial U^{\star} / \partial n_{E}\right] \quad \text { on } E \cap \gamma_{\omega}, E \in \mathcal{E}_{\Omega}
$$

Therefore,

$$
\begin{aligned}
\int_{\gamma_{N}^{\star}} z^{\star} \partial U^{\star} / \partial n_{\omega} d s-\int_{\gamma_{\omega}} z \partial U / \partial n d s-\int_{(\partial \omega) \cap\left(\cup \mathcal{E}_{\Omega}\right)} z^{\star}\left[\partial U^{\star} / \partial n_{\mathcal{E}}\right] d s \\
\quad=\int_{\partial \omega} z^{\star} \partial U^{\star} / \partial n_{\omega} d s \\
=\sum_{j=1}^{J} \int_{\partial \omega_{j}} z^{\star} \partial U_{j}^{\star} / \partial n_{\omega_{j}} d s+\int_{\left(\cup \mathcal{E}_{\Omega}\right) \cap \omega} z^{\star}\left[\partial U^{\star} / \partial n_{\mathcal{E}}\right] d s .
\end{aligned}
$$

We used $z^{\star}=0$ on $\gamma_{D}^{\star}$ and that the definition of the jumps $\left[\partial U^{\star} / \partial n_{\mathcal{E}}\right]$ does not depend on the underlying orientation of $n_{\mathcal{E}}$. The combination of the last four identities shows

$$
\begin{aligned}
\int_{\gamma_{\omega}} z \partial U / \partial n d s=-\int_{\left(\gamma_{\omega} \cup \omega\right) \cap(\cup \mathcal{E})} z^{\star}\left[\partial U^{\star} / \partial n_{\mathcal{E}}\right] d s+\int_{\gamma_{N}^{\star}} z^{\star} \partial U^{\star} / \partial n_{\omega} d s \\
+\sum_{j=1}^{J} \int_{\partial \omega_{j}}\left(c_{\omega}-z^{\star}\right) \partial U^{\star} / \partial n_{\omega_{j}} d s .
\end{aligned}
$$


A summation over all holes $\omega \in \mathcal{C}$ and a rearrangement of boundary pieces of $\cup\left\{\partial \omega_{j}: j=1, \ldots, J\right\}$ yield

$$
\begin{gathered}
\int_{\gamma} z \frac{\partial U}{\partial n} d s=-\int_{\left(\Omega^{\star} \backslash \Omega\right) \cap(\cup \mathcal{E})} z^{\star}\left[\frac{\partial U^{\star}}{\partial n_{\mathcal{E}}}\right] d s+\int_{\Gamma_{N}^{\star} \backslash \Gamma_{N}} z^{\star} \frac{\partial U^{\star}}{\partial n} d s \\
+\sum_{\omega \in \mathcal{C}}\left(\int_{\partial \omega}\left(c_{\omega}-z^{\star}\right) \frac{\partial U^{\star}}{\partial n_{\omega}} d s+\int_{\omega \cap(\cup \mathcal{E})}\left(c_{\omega}-z^{\star}\right)\left[\frac{\partial U^{\star}}{\partial n_{\mathcal{E}}}\right] d s\right) .
\end{gathered}
$$

Combining this representation with (5.5) leads to

$$
\begin{aligned}
\int_{\Omega} \nabla & \cdot \nabla z d x=\int_{\cup \mathcal{E}_{\Omega}}\left[\partial U^{\star} / \partial n_{\mathcal{E}}\right] z^{\star} d s \\
& +\int_{\Gamma_{N}^{\star}}\left(g^{\star}-\partial U^{\star} / \partial n\right) z d s+\int_{\Omega^{\star}} f^{\star} z^{\star} d x \\
& +\sum_{\omega \in \mathcal{C}}\left(\int_{\partial \omega}\left(z^{\star}-c_{\omega}\right) \frac{\partial U^{\star}}{\partial n_{\omega}} d s+\int_{\omega \cap(\cup \mathcal{E})}\left(z^{\star}-c_{\omega}\right)\left[\frac{\partial U^{\star}}{\partial n_{\mathcal{E}}}\right] d s\right) .
\end{aligned}
$$

The first three summands on the right-hand side of (5.9) can be estimated with standard arguments (e.g., from [V]) utilising (5.2) and Cauchy's inequality. The last contribution of $(5.9)$ is bounded by

$$
\sum_{\omega \in \mathcal{C}} h_{\omega}^{-1 / 2}\left\|z^{\star}-c_{\omega}\right\|_{L^{2}(\omega \cap(\cup \mathcal{E}))} h_{\omega}^{1 / 2}\left\|\left[\frac{\partial U^{\star}}{\partial n_{\mathcal{E}}}\right]\right\|_{L^{2}(\omega \cap(\cup \mathcal{E}))}
$$

The trace inequality (cf. Lemma 4.2), (5.1), and a Poincaré inequality with proper $c_{\omega}$ (cf. Subsection 4.4 ) result in

$$
\begin{aligned}
\left\|z^{\star}-c_{\omega}\right\|_{L^{2}(\omega \cap(\cup \mathcal{E}))}^{2} & \lesssim h_{\omega}^{-1}\left\|z^{\star}-c_{\omega}\right\|_{L^{2}\left(V_{\omega}\right)}^{2}+h_{\omega}\left|z^{\star}\right|_{H^{1}\left(V_{\omega}\right)}^{2} \\
& \lesssim h_{\omega}\left|z^{\star}\right|_{H^{1}\left(V_{\omega}\right)}^{2} .
\end{aligned}
$$

Its combination with (5.10) yields (recall the finite overlap of the neighbourhoods $V_{\omega}$ from (5.1)) and the boundedness of the extension operator)

$$
\sum_{\omega \in \mathcal{C}} \int_{\omega \cap(\cup \mathcal{E})}\left(z^{\star}-c_{\omega}\right)\left[\partial U^{\star} / \partial n_{\mathcal{E}}\right] d s \lesssim \eta_{\mathcal{E}}\left|z^{\star}\right|_{H^{1}\left(\Omega^{\star}\right)}
$$

For the second last term on the right-hand side of (5.9), we consider first the case that $\left|\Gamma_{D}^{\star} \cap \partial \omega\right|=0$ and employ analogous arguments to obtain

$$
\sum_{\omega \in \mathcal{C}} \int_{\partial \omega}\left(z^{\star}-c_{\omega}\right) \frac{\partial U^{\star}}{\partial n_{\omega}} d s \lesssim \eta_{\mathcal{C}}\left|z^{\star}\right|_{H^{1}\left(\Omega^{\star}\right)}
$$

since $\partial \omega \backslash \Gamma_{D}^{\star}$ equals $\partial \omega$ up to a set of measure zero (cf. (3.3)). If $\left|\Gamma_{D}^{\star} \cap \partial \omega\right|>0$ we set $c_{\omega}=0$. Employing $z^{\star}=0$ on $\Gamma_{D}^{\star}$ and the trace 
theorem (cf. Section 4.3) yield

$$
\begin{aligned}
\int_{\partial \omega}\left(z^{\star}-c_{\omega}\right) \partial U^{\star} / \partial n_{\omega} d s & \lesssim\left\|\partial U^{\star} / \partial n\right\|_{L^{2}\left(\partial \omega \backslash \Gamma_{D}^{\star}\right)} \\
& \times\left(h_{\omega}^{-1}\left\|z^{\star}\right\|_{L^{2}\left(V_{\omega}\right)}^{2}+h_{\omega}\left|z^{\star}\right|_{H^{1}\left(V_{\omega}\right)}^{2}\right)^{1 / 2} .
\end{aligned}
$$

Since $\left|\partial \omega \cap \Gamma_{D}^{\star}\right|>0$ and using again $\left.z^{\star}\right|_{\Gamma_{D}^{\star}}=0$, a Friedrichs' inequality leads to $\left\|z^{\star}\right\|_{L^{2}\left(V_{\omega}\right)}^{2} \lesssim h_{\omega}\left|z^{\star}\right|_{H^{1}\left(V_{\omega}\right)}^{2}$ and

$$
\sum_{\omega \in \mathcal{C}} \int_{\partial \omega}\left(z^{\star}-c_{\omega}\right) \frac{\partial U^{\star}}{\partial n_{\omega}} d s \lesssim \eta_{\mathcal{C}}\left|z^{\star}\right|_{H^{1}\left(\Omega^{\star}\right)} .
$$

The combination of the above estimates concludes the proof of Theorem 3.1.

\section{Efficiency: Geometric Preliminaries and Main Result}

This section is devoted to the presentation of sufficient assumptions for the converse (called efficiency) estimate of Theorem 3.1 and so to the sharpness of that (reliability) estimate. For the ease of this discussion, we assume throughout this section that all holes are compactly embedded in $\Omega^{\star}$. Otherwise, the hole boundaries $(\partial \omega) \cap \Gamma_{D}^{\star}$ and $(\partial \omega) \cap \Gamma_{N}^{\star}$ would require a special treatment, i.e., a modification of the extension operator $\mathfrak{E}_{D}$. The main part of this section is devoted to characterise a class of holes (of quite general geometry) that allows for an efficiency estimate. The main result is stated in Theorem 6.1 and proved in the subsequent section.

Assumption 6.1. For any hole $\omega \in \mathcal{C}$, the neighbourhood $V_{\omega}$ from Notation 4.1 is compactly included in $\Omega^{\star}$.

Remark 6.1. The definition of $V_{\omega}$ could be generalised by replacing the factor 2 in Notation 4.1 by any other factor which is larger than one or even to more generally shaped neighbourhoods $V_{\omega}$, where $\partial V_{\omega}$ has positive distance to $\omega$.

Definition 6.1. For any ball $B$ let $\rho_{B}$ be the standard mollifier $\rho_{B} \in$ $\mathcal{D}(B)$ with $0 \leq \rho_{B} \leq 1$, i.e., for the ball $B$ around $z$ with radius $r>0$,

$$
\rho_{B}(x)=\exp \left(1 / r+1 /\left(|x-z|^{2}-r\right)\right) \quad \text { if } x \in B \text { and } \rho_{B}(x)=0 \text { else. }
$$

Assumption 6.2. Suppose that, for any edge $E \in \mathcal{E}$, there exists a ball $B_{E} \subset \Omega^{\star}$ with $E \cap B_{E}=\emptyset$ and
(a) $\operatorname{diam}\left(B_{E}\right) \approx h_{E}$
(b) $\operatorname{dist}\left(B_{E}, E\right) \lesssim h_{E}$
(size control), (distance control), 
(c) $\int_{\Omega} \rho_{B_{E}} d x \approx h_{E}^{2} \quad$ (porosity control).

Let $\sigma_{E}$ denote the union of $B_{E}$ with all triangles $T \in \mathcal{T}$ with $E \subset T$.

Assumption 6.3. Suppose that, for any element $T \in \mathcal{T}$, there exists a ball $B_{T} \subset \Omega^{\star} \backslash(\cup \mathcal{E})$ with
(a) $\operatorname{diam}\left(B_{T}\right) \approx h_{T}$
(b) $\operatorname{dist}\left(B_{T}, T\right) \lesssim h_{T}$
(c) $\int_{\Omega} \rho_{B_{T}} d x \approx \tilde{h}_{T}^{2}$
(size control), (distance control), (porosity control).

Set $\sigma_{T}:=T \cup B_{T}$.

The local efficiency estimate for the contribution of the hole $\omega \in \mathcal{C}$ requires a set of additional assumptions.

First, we will mollify and extend the normal $n$ along $\partial \omega$ (that points into $\left.\omega ; n=-n_{\omega}\right)$ to a neighbourhood of $\partial \omega$. Some examples in Figure 5 illustrate Assumption 6.4.

Assumption 6.4. Suppose that, for each hole $\omega \in \mathcal{C}$, there exists $q=q(\omega) \in \mathbb{N}$ disjoint curves $\gamma_{1}, \ldots, \gamma_{q}$ and open balls $B_{1}, \ldots, B_{q}$ so that

(a) $\omega$ is a Lipschitz domain (global smoothness),

(b) $\partial \omega=\overline{\gamma_{1} \cup \cdots \cup \gamma_{q}}$ and $\gamma_{1}, \ldots, \gamma_{q}$ are $C^{2}$ (local smoothness),

(c) the orthogonal projection $P: B \rightarrow \partial \omega$ (projection property), is unique on $B:=\overline{B_{1} \cup \cdots \cup B_{q}}$

$\left.\begin{array}{l}\text { (d1) } B_{j} \cap \gamma_{j} \neq \emptyset, \\ \text { (d2) } B_{j} \cap \gamma_{j} \text { has positive distance to } \partial \omega \backslash \gamma_{j}, \\ \text { (d3) } \overline{B_{1}}, \ldots, \overline{B_{q}} \text { are pairwise disjoint, } \\ \text { (d4) } h_{\omega} \sim\left|\gamma_{j}\right| \sim|\partial \omega| \sim\left|B_{j} \cap V_{\omega}\right|^{1 / 2},\end{array}\right\} \quad\left(\begin{array}{l}\text { distortion } \\ \text { control }\end{array}\right)$.

Introduce the extended domain $\Omega^{\star \star}$, the intersections $V_{\omega}^{\text {int }}$ and $V_{\omega}^{\star \star}$ with $B_{j}(\omega)$ and $q(\omega)$ as in Assumption 6.4 (cf. Figure 6, 7 for an illustration) by

$$
\Omega^{\star \star}:=\Omega \cup \sum_{\omega \in \mathcal{C}} \sum_{j=1}^{q(\omega)} B_{j}(\omega), \quad V_{\omega}^{i n t}:=V_{\omega} \cap \Omega, \quad V_{\omega}^{\star \star}:=V_{\omega} \cap \Omega^{\star \star}
$$

Since $f \in L^{2}(\Omega)$, the solution of the differential equation belongs to the Hilbert space $H_{L}^{1}(\Omega):=\left\{u \in H^{1}(\Omega): \Delta u \in L^{2}(\Omega)\right\}$ with

$$
(u, v)_{H_{L}^{1}(\Omega)}:=(u, v)_{H^{1}(\Omega)}+(\Delta u, \Delta v)_{L^{2}(\Omega)}, \quad\|u\|_{H_{L}^{1}(\Omega)}:=(u, u)_{H_{L}^{1}(\Omega)}^{1 / 2} .
$$



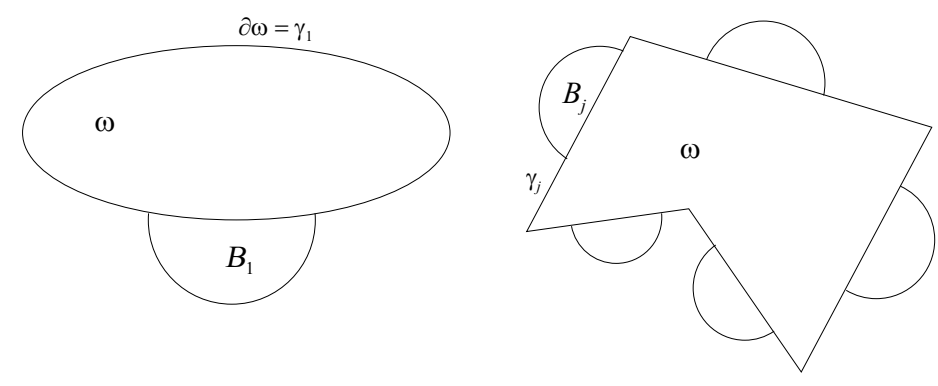

$\omega$

Figure 5. The domains (a) and (b) satisfy Assumption 6.4 with moderate constants in the estimates while this is not the case for domain (c).

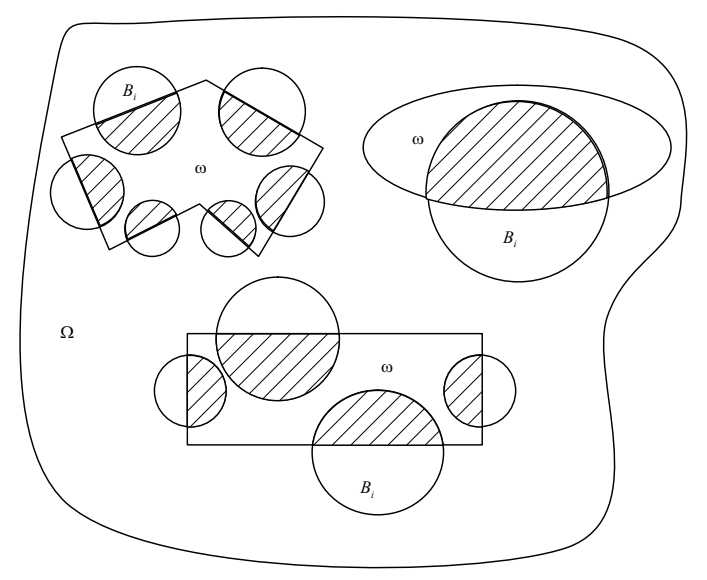

FiguRE 6. Domain $\Omega$ with holes. The extended domain $\Omega^{* *}$ arises by including the shaded half balls to $\Omega$.

The following assumption concerns the existence of an extension operator for a subspace of $H_{L}^{1}(\Omega)$. Let us introduce

$$
\mathcal{W}(\Omega):=\left\{u \in H_{L}^{1}(\Omega): \partial u / \partial n=0 \text { on } \gamma\right\} \oplus \mathcal{S}
$$

equipped with the $H_{L}^{1}(\Omega)$-norm.

Assumption 6.5. Adopt Assumption 6.4 and suppose

(a) there exists a continuous extension operator $\mathfrak{E}_{L}: \mathcal{W}(\Omega) \rightarrow$ $H_{L}^{1}\left(\Omega^{\star \star}\right)$ so that, for all $u \in \mathcal{W}(\Omega)$ and $\omega \in \mathcal{C}$,

$$
\left\|\mathfrak{E}_{L} u\right\|_{H_{L}^{1}\left(V_{\omega}^{\star \star}\right)} \leq c_{16}\|u\|_{H_{L}^{1}\left(V_{\omega}^{i n t}\right)} .
$$




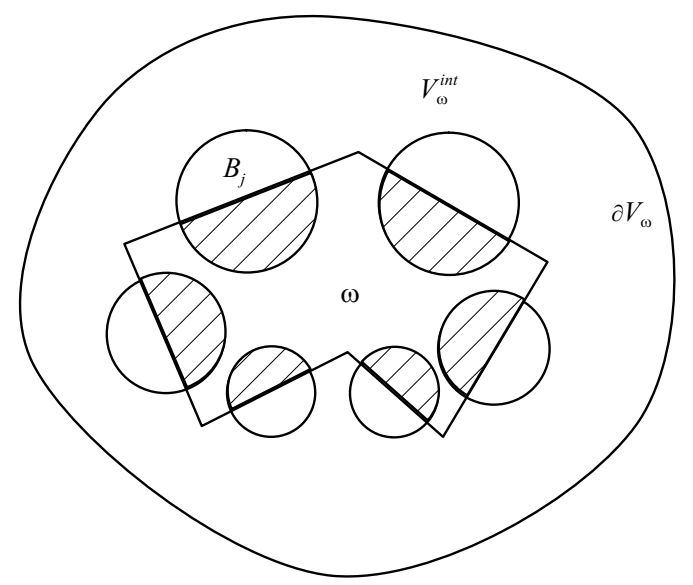

FiguRE 7 . Neighborhood $V_{\omega}$ of $\omega$ with boundary $\partial V_{\omega}$, intersection $V_{\omega}^{\text {int }}=V_{\omega} \cap \Omega$ and extended intersection $V_{\omega}^{* *}=V_{\omega} \cap \Omega^{* *}$.

(b) If $u \in \mathcal{W}(\Omega)$ and $\Delta u \in H^{1}\left(V_{\omega}^{i n t}\right)$, then, $\Delta \mathfrak{E}_{L} u \in H^{1}\left(V_{\omega}^{\star \star}\right)$ and

$$
\left\|\Delta \mathfrak{E}_{L} u\right\|_{H^{1}\left(V_{\omega}^{\star \star}\right)} \leq c_{17}\|\Delta u\|_{H^{1}\left(V_{\omega}^{i n t}\right)},
$$

(c) if $u$ is affine on some $B_{j}(\omega) \cap \Omega$, then, the extension $\mathfrak{E}_{L} u$ is the affine extension on $B_{j}(\omega)$.

Remark 6.2. Assumption 6.5 implies that, for all $u \in \mathcal{W}(\Omega)$ and $v \in$ $C_{0}^{\infty}\left(B_{j}(\omega)\right)$

$$
\int_{B_{j}(\omega)} \nabla v \cdot \nabla\left(\mathfrak{E}_{L} u\right)-v \Delta\left(\mathfrak{E}_{L} u\right) d x=0
$$

Remark 6.3. Consider $\Omega=(-1,0) \times(0,1)$ and $\Omega^{\star}=(-1,1) \times(0,1)$ with the hole $\omega=\Omega^{\star} \backslash \bar{\Omega}$ and the inner boundary $\gamma:=\bar{\Omega} \cap \overline{\Omega^{\star}}$. Then, there exists no extension operator from $H_{L}^{1}(\Omega)$ into $H_{L}^{1}\left(\Omega^{\star}\right)$. The reason is that all functions $w \in H_{L}^{1}\left(\Omega^{\star}\right)$ satisfy $w \in H_{l o c}^{2}\left(\Omega^{\star}\right)$ and, hence, $\left.w\right|_{\gamma} \in$ $H_{l o c}^{3 / 2}(\gamma)$, while the trace map $t r: H^{1}(\Omega) \rightarrow H^{1 / 2}(\gamma)$ is surjective. As an example, the function $u \in H_{L}^{1}(\Omega)$ defined by $u(x)=r^{\lambda} \sin (\lambda \varphi)$, where $(r, \varphi)$ are polar coordinates in $\Omega$ centred at $(0,1 / 2)$ and $\lambda \in$ $(0,1)$, belongs to $H_{L}^{1}(\Omega)$ and cannot be extended to $u^{\star} \in H_{L}^{1}\left(\Omega^{\star}\right){ }^{1}$

The extension operator $\mathfrak{E}_{L}$ is constructed for one typical polygonal hole.

\footnotetext{
${ }^{1}$ Thanks are due to M. Costabel for providing us with this Remark.
} 
Example 6.1. Let $\Omega, \Omega^{\star}, \omega, \gamma$ be as in Remark 6.3. Then, there exists an extension operator $\mathfrak{E}_{L}: \mathcal{W}(\Omega) \rightarrow H_{L}^{1}\left(\Omega^{\star}\right)$ which satisfies Assumption 6.5. For $u \in H_{L}^{1}(\Omega)$, the extended function $u^{\star}:=\mathfrak{E}_{L} u$ is given, for $x \in \omega$, by

$$
u^{\star}\left(x_{1}, x_{2}\right):=u\left(-x_{1}, x_{2}\right)+2 x_{1} \frac{\partial u}{\partial x_{1}}\left(0, x_{2}\right) .
$$

For $u \in \mathcal{W}(\Omega)$ and $x \in \Omega$, let

$$
u_{2}(x):=x_{1} \frac{\partial u}{\partial x_{1}}\left(0, x_{2}\right) .
$$

The definition of $\mathcal{W}(\Omega)$ implies that $\partial u / \partial x_{1}$ is constant on $\gamma$ and, hence, $u_{2} \in \mathcal{S}$. The function $u_{1}:=u-u_{2}$ satisfies $\partial u_{1} / \partial x_{1}=0$ on $\gamma$. The linearity of $\mathfrak{E}_{L}$ allows to investigate $u_{1}^{\star}:=\mathfrak{E}_{L} u_{1}$ and $u_{2}^{\star}:=\mathfrak{E}_{L} u_{2}$ separately. For $u_{1}$ and $\left(x_{1}, x_{2}\right) \in \omega$, the extension operator simplifies to $u_{1}^{\star}\left(x_{1}, x_{2}\right):=u_{1}\left(-x_{1}, x_{2}\right)$. The density of $C^{\infty}(\Omega) \cap H_{L}^{1}(\Omega)$ in $H_{L}^{1}(\Omega)$ implies that it is sufficient to investigate the boundedness of $\mathfrak{E}_{L} u_{1}$ for functions $u_{1} \in C^{\infty}(\Omega) \cap \mathcal{W}(\Omega)$. Simple calculations result in $u_{1}^{\star}=$ $u_{1}, \nabla u_{1}^{\star}=\nabla u_{1}$ on $\gamma$ and $\Delta\left(\mathfrak{E}_{L} u_{1}\right)\left(x_{1}, x_{2}\right)=\left(\Delta u_{1}\right)\left(-x_{1}, x_{2}\right)$ on $\omega$. Consequently, for all $u_{1}^{\star} \in H_{L}^{1}(\Omega)$, there holds

$$
\left\|u_{1}^{\star}\right\|_{H_{L}^{1}\left(\Omega^{\star}\right)}=2\left\|u_{1}\right\|_{H_{L}^{1}(\Omega)} .
$$

If, in addition, $\Delta u_{1}^{\star} \in H^{1}\left(\Omega^{\star}\right)$, then,

$$
\left\|\Delta u_{1}^{\star}\right\|_{H_{L}^{1}\left(\Omega^{\star}\right)}=2\left\|\Delta u_{1}\right\|_{H_{L}^{1}(\Omega)} .
$$

The definition (6.3) directly implies that affine functions are extended analytically, i.e., "by themselves". The proof of

$$
\left\|u_{2}^{\star}\right\|_{H_{L}^{1}\left(\Omega^{\star}\right)} \lesssim\left\|u_{2}\right\|_{H_{L}^{1}(\Omega)}, \quad\left\|\Delta u_{1}^{\star}\right\|_{L^{2}\left(\Omega^{\star}\right)}=\left\|\Delta u_{1}\right\|_{L^{2}(\Omega)}=0
$$

is straightforward. Next,

$$
\left\|u_{2}\right\|_{H_{L}^{1}(\Omega)} \lesssim\left\|\partial u / \partial x_{1}\right\|_{H^{-1 / 2}(\gamma)}
$$

The continuity of the trace operators in $H_{L}^{1}(\Omega)$ implies $\left\|u_{2}\right\|_{H_{L}^{1}(\Omega)} \lesssim$ $\|u\|_{H_{L}^{1}(\Omega)}$ from which we conclude

$$
\begin{aligned}
\left\|u^{\star}\right\|_{H_{L}^{1}\left(\Omega^{\star}\right)} & \lesssim\left\|u_{1}\right\|_{H_{L}^{1}(\Omega)}+\left\|u_{2}\right\|_{H_{L}^{1}(\Omega)} \\
& \leq\|u\|_{H_{L}^{1}(\Omega)}+2\left\|u_{2}\right\|_{H_{L}^{1}(\Omega)} \lesssim\|u\|_{H_{L}^{1}(\Omega)} .
\end{aligned}
$$


To mollify and to extend the normal field $n$ to some neighbourhood of the hole boundaries we employ the ansatz (recall the definition of $B$ from Assumption 6.4)

$$
N= \begin{cases}\lambda n \circ P & \text { in } B, \\ 0 & \text { otherwise. }\end{cases}
$$

The function $\lambda$ is a generalization of bubble functions from the a posteriori error analysis $[\mathrm{V}]$ with an integral mean orthogonal to the direction $(\cos \alpha, \sin \alpha)$.

Assumption 6.6. The function $\lambda=\lambda_{\alpha} \in C^{\infty}\left(\mathbb{R}^{2}\right)$ in (6.4) depends continuously on $\alpha \in[-\pi, \pi]$ and satisfies

(a) $\left.\begin{array}{c}\operatorname{supp} \lambda_{\alpha} \subseteq \bar{B} \text { and } 0 \leq \lambda_{\alpha} \leq 1 \text { on } \partial \omega \\ \left|\lambda_{\alpha}\right|_{L^{\infty}\left(\mathbb{R}^{2}\right)}+h_{\omega}\left|\lambda_{\alpha}\right|_{W^{1, \infty}\left(\mathbb{R}^{2}\right)} \lesssim 1\end{array}\right\} \quad$ (cut-off function),

$\left.\begin{array}{l}\text { (b) any } \gamma_{j} \text { there exists a sub-arc } \widetilde{\gamma}_{j} \subset \gamma_{j} \\ \text { with }\left|\gamma_{j}\right| \sim\left|\widetilde{\gamma}_{j}\right| \text { and } \lambda_{\alpha} \geq 1 / 2 \text { on } \tilde{\gamma}_{j}\end{array}\right\} \quad$ (positivity),

(c) $\int_{V_{\omega}^{\text {int }}}\left(\begin{array}{l}\cos \alpha \\ \sin \alpha\end{array}\right) \cdot N d x=0 \quad$ ( $\alpha$-orthogonality).

Remark 6.4. The compactness of $[-\pi, \pi]$ and the continuous dependence of $\lambda$ on $\alpha$ imply an $\alpha$-uniform estimate in Assumption 6.6.(a).

We need an abstract assumption on the hole boundaries.

Assumption 6.7. The mollified and extended normal field $N$ is of the form (6.4) where $\lambda$ satisfies Assumption 6.6 and, for all $q \in \mathbb{R}^{2}$,

$$
\begin{aligned}
& h_{\omega}^{-1}\|q \cdot N\|_{L^{2}\left(V_{\omega}\right)}^{2}+h_{\omega}|q \cdot N|_{H^{1}\left(V_{\omega}\right)}^{2} \\
& \lesssim\|q \cdot n\|_{L^{2}(\partial \omega)}^{2} \lesssim \int_{\partial \omega}(q \cdot N)(q \cdot n) d s .
\end{aligned}
$$

We illustrate these abstract assumptions with two typical examples.

Example 6.2. (polygonal hole). Assume that $\omega$ is a polygonal hole satisfying Assumption 6.4 with straight lines $\gamma_{j}$ orthogonal to $n_{j}$. Then,

$$
\begin{aligned}
\int_{V_{\omega}}|q \cdot N|^{2} d x & =\sum_{j=1}^{q} \int_{B_{j} \cap V_{\omega}}|q \cdot \lambda n \circ P|^{2} d x \\
& =\sum_{j=1}^{q}\left|q \cdot n_{j}\right|^{2} \int_{B_{j} \cap V_{\omega}} \lambda^{2} d x \lesssim \sum_{j=1}^{q}\left|q \cdot n_{j}\right|^{2}\left|B_{j} \cap V_{\omega}\right| \\
& =\sum_{j=1}^{q} \frac{\left|B_{j} \cap V_{\omega}\right|}{\left|\gamma_{j}\right|} \int_{\gamma_{j}}\left|q \cdot n_{j}\right|^{2} d s \lesssim h_{\omega} \int_{\partial \omega}|q \cdot n|^{2} d x
\end{aligned}
$$




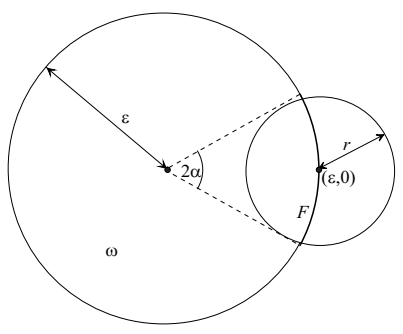

Figure 8. Circular hole $\omega$ in Example 6.3.

The estimate of the $H^{1}$-seminorm follows analogously by using

$$
\int_{B_{i} \cap V_{\omega}}\|\nabla \lambda\|^{2} d x \lesssim 1 .
$$

The second inequality in (6.5) follows from

$$
\begin{aligned}
\int_{\partial \omega}|q \cdot n|^{2} d x & =\sum_{j=1}^{q}\left|q \cdot n_{j}\right|^{2} \frac{\left|\gamma_{j}\right|}{\left|\widetilde{\gamma}_{j}\right|} \int_{\widetilde{\gamma}_{j}} 1 d s \\
& \lesssim \sum_{j=1}^{q}\left|q \cdot n_{j}\right|^{2} \int_{\widetilde{\gamma}_{j}} \lambda d s \leq \int_{\partial \omega}(q \cdot N)(q \cdot n) d s
\end{aligned}
$$

Remark 6.5. The estimates in Example 6.2 are based on Assumptions 6.4 and 6.6 and so are the multiplicative constants hidden in the notation $\lesssim$.

Remark 6.6. The condition (6.5) is partly redundant as $\|q \cdot N\|_{L^{2}\left(V_{\omega}\right)}$ is bounded by $\operatorname{diam}\left(V_{\omega}\right)|q \cdot N|_{H^{1}\left(V_{\omega}\right)}$ owing to a Friedrichs inequality.

The following example shows that Assumption 6.7 may hold with moderate constant for holes with curved boundary (cf. Figure 8).

Example 6.3 (circle). If $\partial \omega$ is a circle and $F$ is a sub-arc of $\partial \omega$ we find polar coordinates centred at the midpoint of $\omega$ and, without loss of generality, suppose $\omega=B(0, \varepsilon)$ and $F=\{\varepsilon(\cos (\varphi, \sin \varphi):-\alpha<$ $\varphi<\alpha\}$ for some $\varepsilon>0$ and $0<\alpha<\pi / 2$. Let $\lambda$ be a scaled mollifier with centre at $(\varepsilon, 0)$ and support $B:=\overline{B((\varepsilon, 0), r)}$ for $r:=$ $\varepsilon \min \{1 / 2, \sqrt{2(1-\cos \alpha)}\}$. then, $N(r, \varphi):=\varrho_{B}(r, \varphi)(\cos \varphi, \sin \varphi)$ satisfies Assumption 6.7. The constant in (6.5) is independent of $\varepsilon$ but degenerates if $\alpha$ is small.

Remark 6.7. The previous two examples illustrate that and how $N$ can be constructed for a quite large class of piecewise smooth domains: Corners are cut-off. It is also clear that the support $V_{\omega}$ of $N$ can be a 


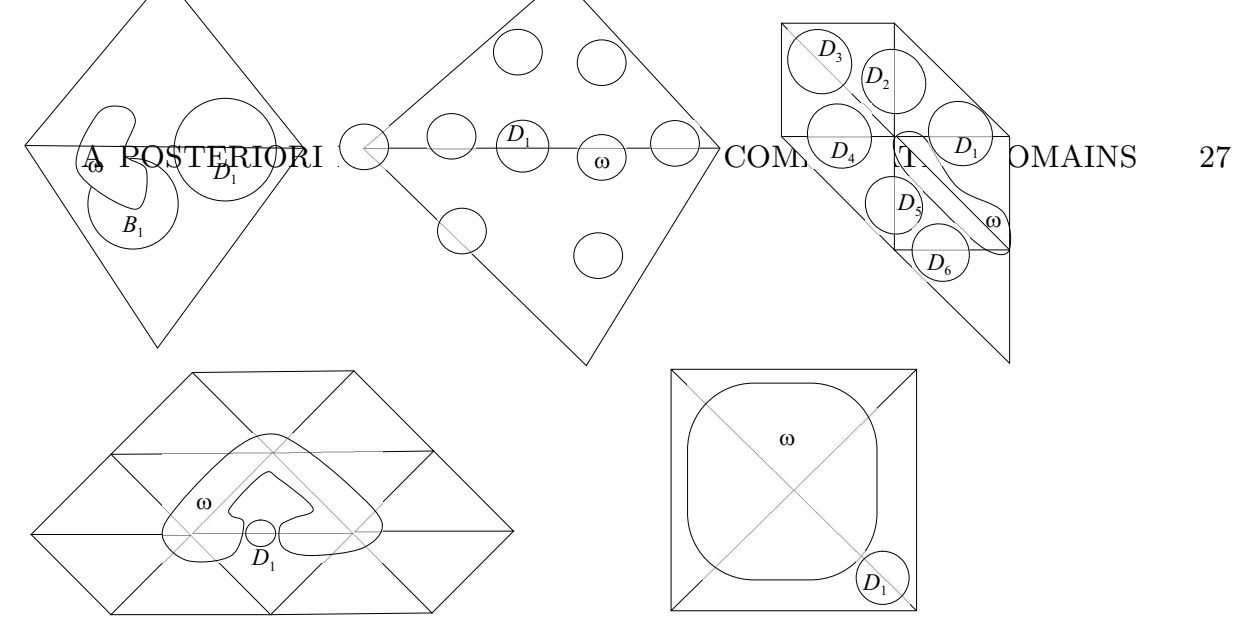

Figure 9. Triangulations covering a hole $\omega$. The balls of connecting edges are denoted by $D_{j}$ while the balls for each smooth component $\gamma_{j}$ are denoted by $B_{j}$

subset of an arbitrary small neighbourhood of $\partial \omega$ on the expense of a large constant in (6.5).

The subsequent notions concern the patch around a hole and allow that holes may intersect arbitrarily with the mesh.

Definition 6.2. Let

$$
\mathcal{T}_{\omega}:=\{T \in \mathcal{T}: T \cap \partial \omega \neq \emptyset\} .
$$

A sequence $\left(T_{j}\right)_{j=0}^{J}$ of triangles in $\mathcal{T}$ is edge-connected if, for $j=$ $1,2, \ldots, J$, the triangles $T_{j-1}, T_{j}$ share a common edge $E_{j}$.

Some characteristic examples illustrating Assumption 6.8 are depicted in Figure 9.

Assumption 6.8. For any hole $\omega \in \mathcal{C}$ and any $K \in \mathcal{T}_{\omega}$ there is a sequence of edge-connected triangles $\left(K_{j}\right)_{j=0}^{J}$ in $\mathcal{T}$ with $J=J(K)$ such that, for all edges $E_{j}=K_{j-1} \cap K_{j}$, there exists a ball $D_{j}$ with radius $r_{j}$ centred at $M_{j} \in E_{j}$ and

(a) $r_{j} \gtrsim h_{\omega}$

(b) $D_{j} \subset \Omega^{\star \star}$,

(c) the endpoints of $E_{j}$ have positive distance to $D_{j} \cap E_{j}$.

Remark 6.8. Assumption 6.8 can be generalized by allowing more general domains $D_{j}$ for connecting neighbouring triangles with finite overlap.

Remark 6.9. The definition of the balls $D_{j}$ in Assumption 6.8 implies that, for all $U^{\star} \in \mathcal{S}^{\star}$ and $U:=\left.U^{\star}\right|_{\Omega}$, there holds $U^{\star}=\mathfrak{E}_{L} U$ on $D_{j}$. 
The constants in the preceding assumptions of this section enter in the multiplicative constant in the efficiency estimate. Its proof is the contents of the next section. Recall the definition of $\sigma_{T}$ and $\sigma_{E}$ from Assumption 6.2 and 6.3.

Theorem 6.1. Under the Assumptions 6.1-6.8 and notation of Section 2 we have with $f_{L}^{\star}:=\Delta \mathfrak{E}_{L} u$

$$
\begin{aligned}
\eta_{\Omega}^{2}+ & \eta_{\mathcal{E}}^{2}+\eta_{\mathcal{C}}^{2} \lesssim\|\nabla e\|_{L^{2}(\Omega)}^{2}+\sum_{E \in \mathcal{E}_{N}} h_{E} \min _{g_{E} \in \mathbb{R}}\left\|\left(g-g_{E}\right)\right\|_{L^{2}\left(E \cap \Gamma_{N}\right)}^{2} \\
& +\sum_{T \in \mathcal{T}} h_{T}^{2} \min _{f_{T} \in \mathbb{R}}\left\|f-f_{T}\right\|_{L^{2}\left(\Omega \cap \sigma_{T}\right)}^{2}+\sum_{E \in \mathcal{E}} h_{E}^{2} \min _{f_{E} \in \mathbb{R}}\left\|f-f_{E}\right\|_{L^{2}\left(\Omega \cap \sigma_{E}\right)}^{2} \\
& +\sum_{\omega \in \mathcal{C}} h_{\omega}^{2} \min _{f_{\omega} \in \mathbb{R}}\left\|f_{L}^{\star}-f_{\omega}\right\|_{L^{2}\left(V_{\omega}^{\star \star}\right)}^{2} .
\end{aligned}
$$

Remark 6.10. Theorem 6.1 even holds in a more local form as shown in the proof in Section 7.

Remark 6.11. The third and fourth term are of higher order if the right-hand side $f$ is smooth in the sense that it is the restriction of a function $F$ in $H^{1}\left(\Omega^{\star}\right)$. Indeed, $f=\left.F\right|_{\Omega}$ yields

$$
\min _{f_{T} \in \mathbb{R}}\left\|f-f_{T}\right\|_{L^{2}\left(\Omega \cap \sigma_{T}\right)} \leq \min _{F_{T} \in \mathbb{R}}\left\|F-F_{T}\right\|_{L^{2}\left(\sigma_{T}\right)} \lesssim h_{T}\|\nabla F\|_{L^{2}\left(\sigma_{T}\right)}
$$

according to (4.7) and Assumption 6.3. An analogous estimate holds for $\min _{f_{E} \in \mathbb{R}}\left\|f-f_{E}\right\|_{L^{2}\left(\Omega \cap \sigma_{E}\right)}$. If $f \in H^{1}\left(V_{\omega}^{\text {int }}\right)$, Assumption 6.5 implies $f_{L}^{\star}=\Delta \mathfrak{E}_{L} u \in H_{L}^{1}\left(V_{\omega}^{\star \star}\right)$ and the last term is of higher order

$$
\begin{aligned}
\min _{f_{\omega} \in \mathbb{R}}\left\|f_{L}^{\star}-f_{\omega}\right\|_{L^{2}\left(V_{\omega}^{\star \star}\right)} & \lesssim h_{\omega}\left\|\Delta \mathfrak{E}_{L} u\right\|_{H^{1}\left(V_{\omega}^{\star \star}\right)} \leq c_{17} h_{\omega}\|\Delta u\|_{H^{1}\left(V_{\omega}^{i n t}\right)} \\
& =c_{17} h_{\omega}\|f\|_{H^{1}\left(V_{\omega}^{i n t}\right)} .
\end{aligned}
$$

The second term is of higher order if there exists $G \in H^{1}\left(\Gamma_{N}^{\star}\right)$ such that $g=\left.G\right|_{\Gamma_{N}}$. In this case, there holds

$$
\sum_{E \in \mathcal{E}_{N}} h_{E} \min _{g_{E} \in \mathbb{R}}\left\|\left(g-g_{E}\right)\right\|_{L^{2}\left(E \cap \Gamma_{N}\right)}^{2} \lesssim\left\|h_{\mathcal{E}}^{3 / 2} \partial G / \partial s\right\|_{L^{2}\left(\Gamma_{N}^{\star}\right)}^{2} .
$$

\section{Proof of Efficiency}

The following results provide local estimates summarised in Theorem 6.1. The combination of Lemma 7.2, 7.4, 7.5, and 7.6 is the proof of the theorem. 
Definition 7.1. For any edge $E$ with ball $B_{E}$ from Assumption 6.2 let $\beta_{E}$ be the piecewise quadratic product of the two barycentric coordinates with $\beta_{E}=\lambda_{1} \lambda_{2}$ on $T \in \mathcal{T}$ with $E \subset \partial T$ that vanishes on $\partial T \backslash E$ and equals $s\left(h_{E}-s\right) / h_{E}^{2}$ on $E$ with respect to the arc-length $s$. Let

$$
b_{E}:=\beta_{E}-c_{E} \rho_{B_{E}} \quad \text { for } \quad c_{E}:=\int_{\Omega} \beta_{E} d x / \int_{\Omega} \rho_{B_{E}} d x \in \mathbb{R} .
$$

Lemma 7.1. Under the Assumption 6.2, the function $b_{E}$ from Definition 7.1 equals $s\left(h_{E}-s\right) / h_{E}^{2}$ on $E$ with respect to the arc-length $s$ and satisfies $\operatorname{supp} b_{E} \subset \overline{\sigma_{E}}$,

$$
\int_{\Omega} b_{E} d x=0, \quad \operatorname{Lip}\left(b_{E}\right) \lesssim 1 / h_{E}, \quad \text { and } \quad\left\|\nabla b_{E}\right\|_{L^{2}\left(\sigma_{E}\right)} \lesssim 1 .
$$

Proof. Since $E \cap B_{E}=\emptyset$ in Assumption $6.2, b_{E}=\beta_{E}$ on $E$ and $\int_{\Omega} b_{E} d x=0$ follows by definition of $c_{E}$. The functions $\rho_{B_{E}}$ and $\beta_{E}$ are Lipschitz with Lipschitz constant $\lesssim 1 / h_{E}$. Hence it remains to verify $0 \leq c_{E} \lesssim 1$. Assumption 6.2.(c) shows $c_{E} \lesssim 1$. The remaining estimate then follows from Assumption 6.2.

Definition 7.2. Let $d=1,2$. For any $d$-dimensional measurable set $V \subset \mathbb{R}^{2}$, let $|\omega \cap V|$ denote the $d$-dimensional measure of $\omega \cap V$ and set

$$
\mathcal{C}_{V}:=\{\omega \in \mathcal{C}:|\omega \cap V|>0\} .
$$

Lemma 7.2. We have, for all $E \in \mathcal{E}_{\Omega}$ and $f_{E} \in \mathbb{R}, \mathcal{E}^{\prime}:=\mathcal{E} \backslash\{E\}$,

$$
\begin{aligned}
& \left\|h_{\mathcal{E}}^{1 / 2}\left[\partial U^{\star} / \partial n_{E}\right]\right\|_{L^{2}(E)}^{2} \lesssim\|\nabla e\|_{L^{2}\left(\Omega \cap \sigma_{E}\right)}^{2}+h_{E}^{2}\left\|f-f_{E}\right\|_{L^{2}\left(\Omega \cap \sigma_{E}\right)}^{2} \\
& \quad+\sum_{\omega \in \mathcal{C}_{\sigma_{E}}} h_{\omega}\left\|\partial U^{\star} / \partial n_{\omega}\right\|_{L^{2}(\partial \omega)}^{2}+\sum_{\omega \in \mathcal{C}_{E}} h_{\omega}\left\|\left[\partial U^{\star} / \partial n_{\mathcal{E}}\right]\right\|_{L^{2}\left(\omega \cap\left(\cup \mathcal{E}^{\prime}\right)\right)}^{2}
\end{aligned}
$$

Proof. Let $J_{E}$ denote the constant $\left[\partial U^{\star} / \partial n_{E}\right]$ and notice that (since $b_{E}$ reads $s\left(h_{E}-s\right) / h_{E}^{2}$ on $\left.E\right)$

$$
h_{E}\left\|J_{E}\right\|_{L^{2}(E)}^{2}=h_{E} / 3 J_{E} \int_{E}\left[\partial U^{\star} / \partial n_{E}\right] b_{E} d s .
$$


The last integral has a representation as in (5.9) with $z^{\star}$ replaced by $b_{E}$, namely,

$$
\int_{E} J_{E} b_{E} d s=\int_{\Omega} \nabla e \cdot \nabla b_{E} d x-\int_{\Omega} f b_{E} d x
$$

$$
+\sum_{\omega \in \mathcal{C}}\left(\int_{\partial \omega}\left(c_{\omega}-b_{E}\right) \frac{\partial U}{\partial n_{\omega}} d s+\int_{\omega \cap(\cup \mathcal{E})}\left[\frac{\partial U^{\star}}{\partial n_{\mathcal{E}}}\right]\left(c_{\omega}-b_{E}\right) d s\right) .
$$

For holes $\omega \in \mathcal{C}$ with $\omega \cap E=\emptyset$, the function $b_{E}$ equals zero on $\omega \cap(\cup \mathcal{E})$ and we set in these cases $c_{\omega}=0$. Thus, the sum $\sum_{\omega \in \mathcal{C}}$ over the last integral in (7.3) reduces to a sum over all $\omega \in \mathcal{C}_{E}$, i.e.,

$$
\sum_{\omega \in \mathcal{C}_{E}} J_{E} \int_{\omega \cap E}\left(c_{\omega}-b_{E}\right) d s+\sum_{\omega \in \mathcal{C}_{E}} \int_{\omega \cap\left(\cup \mathcal{E}^{\prime}\right)}\left[\frac{\partial U^{\star}}{\partial n_{\mathcal{E}}}\right]\left(c_{\omega}-b_{E}\right) d s .
$$

For the remaining holes $\omega \in \mathcal{C}_{E}$, we choose the constant $c_{\omega}$ such that $\int_{\omega \cap E}\left(c_{\omega}-b_{E}\right) d s$ vanishes. Then, $c_{\omega}$ equals $b_{E}(\xi)$ for some $\xi$ in the convex hull of $\omega \cap E$ and for any $x \in \omega$ we have $|x-\xi| \lesssim h_{\omega}$. By Lemma 7.1, $b_{E}$ is Lipschitz with $\operatorname{Lip}\left(b_{E}\right) \lesssim 1 / h_{E}$. This and a Cauchy inequality for the length $|\partial \omega|$ show

$$
\begin{aligned}
\int_{\partial \omega}\left|c_{\omega}-b_{E}\right|\left|\partial U^{\star} / \partial n_{\omega}\right| d s & \leq\left\|c_{\omega}-b_{E}\right\|_{L^{2}(\partial \omega)}\left\|\partial U^{\star} / \partial n_{\omega}\right\|_{L^{2}(\partial \omega)} \\
& \lesssim|\partial \omega|^{1 / 2} h_{\omega} / h_{E}\left\|\partial U^{\star} / \partial n_{\omega}\right\|_{L^{2}(\partial \omega)} .
\end{aligned}
$$

The sum $\sum_{\omega \in \mathcal{C}}$ for the second last integral in (7.3) reduces to $\sum_{\omega \in \mathcal{C}_{\sigma_{E}}}$. Note that $\sum_{\omega \in \mathcal{C}_{\sigma_{E}}} h_{\omega}^{2} \lesssim h_{E}^{2}$ and, thus,

$$
\sum_{\omega \in \mathcal{C}} \int_{\partial \omega}\left|c_{\omega}-b_{E}\right|\left|\partial U^{\star} / \partial n_{\omega}\right| d s \leq\left(\sum_{\omega \in \mathcal{C}_{\sigma_{E}}} h_{\omega}\left\|\partial U^{\star} / \partial n_{\omega}\right\|_{L^{2}(\partial \omega)}^{2}\right)^{1 / 2} .
$$

In the same fashion, we obtain by using $\left|\omega \cap\left(\cup \mathcal{E}^{\prime}\right)\right| \lesssim h_{\omega}$ and Cauchy's inequality the estimate

$$
\begin{aligned}
\sum_{\omega \in \mathcal{C}_{E}} \int_{\omega \cap\left(\cup \mathcal{E}^{\prime}\right)}\left|\left[\frac{\partial U^{\star}}{\partial n_{\mathcal{E}}}\right]\left(c_{\omega}-b_{E}\right)\right| d s & \leq \sum_{\omega \in \mathcal{C}_{E}} \frac{h_{\omega}}{h_{E}}\left\|h_{\omega}^{1 / 2}\left[\frac{\partial U^{\star}}{\partial n_{\mathcal{E}}}\right]\right\|_{L^{2}\left(\omega \cap\left(\cup \mathcal{E}^{\prime}\right)\right)} \\
& \leq\left(\sum_{\omega \in \mathcal{C}_{E}}\left\|h_{\omega}^{1 / 2}\left[\frac{\partial U^{\star}}{\partial n_{\mathcal{E}}}\right]\right\|_{L^{2}\left(\omega \cap\left(\cup \mathcal{E}^{\prime}\right)\right)}^{2}\right)^{1 / 2}
\end{aligned}
$$


By construction, $\int_{\Omega} b_{E} d x=0$. Hence, $\int_{\Omega} f b_{E} d x$ can be replaced by $\int_{\Omega}\left(f-f_{E}\right) b_{E} d x$. Taking into account (7.1) we are led to

$$
\begin{aligned}
& \int_{E} J_{E} b_{E} d s \leq\|\nabla e\|_{L^{2}\left(\Omega \cap \sigma_{E}\right)}+\left\|h_{\mathcal{T}}\left(f-f_{E}\right)\right\|_{L^{2}\left(\Omega \cap \sigma_{E}\right)} \\
& +\left(\sum_{\omega \in \mathcal{C}_{\sigma_{E}}}\left\|\frac{\partial U^{\star}}{\partial n_{\omega}}\right\|_{L^{2}(\partial \omega)}^{2}\right)^{1 / 2}+\left(\sum_{\omega \in \mathcal{C}_{E}}\left\|h_{\omega}^{1 / 2}\left[\frac{\partial U^{\star}}{\partial n_{\mathcal{E}}}\right]\right\|_{L^{2}\left(\omega \cap\left(\cup \mathcal{E}^{\prime}\right)\right)}^{2}\right)^{1 / 2} .
\end{aligned}
$$

This concludes the proof.

Definition 7.3. For any triangle $T$ with ball $B_{T}$ from Assumption 6.3 let $\beta_{T}$ be the cubic bubble function which is the product of the three barycentric coordinates, $\beta_{T}=\lambda_{1} \lambda_{2} \lambda_{3}$, on $T$ that vanishes on $\partial T$. Let

$$
b_{T}:=\beta_{T}-c_{T} \rho_{B_{T}} \quad \text { for } \quad c_{T}:=\int_{\Omega} \beta_{T} d x / \int_{\Omega} \rho_{B_{T}} d x \in \mathbb{R} .
$$

Lemma 7.3. Under the Assumption 6.3, the function $b_{T}$ from Definition 7.3 satisfies $\operatorname{supp} b_{T} \subset \overline{\sigma_{T}}$, and

$$
\int_{\Omega} b_{T} d x=0, \quad \operatorname{Lip}\left(b_{T}\right) \lesssim 1 / h_{T}, \quad \text { and } \quad\left\|\nabla b_{T}\right\|_{L^{2}\left(\sigma_{T}\right)} \lesssim 1 .
$$

Proof. The proof is analogous to that of Lemma 7.1 and so omitted.

Lemma 7.4. We have, for all $T \in \mathcal{T}$ and $f_{T} \in \mathbb{R}$,

$$
\begin{aligned}
&\left\|h_{T} f\right\|_{L^{2}(\Omega \cap T)}^{2} \lesssim\|\nabla e\|_{L^{2}\left(\Omega \cap \sigma_{T}\right)}^{2} \\
& \quad+h_{T}^{2}\left\|f-f_{T}\right\|_{L^{2}\left(\Omega \cap \sigma_{T}\right)}^{2}+\sum_{\omega \in \mathcal{C}_{\sigma_{T}}} h_{\omega}\left\|\partial U^{\star} / \partial n_{\omega}\right\|_{L^{2}(\partial \omega)}^{2} .
\end{aligned}
$$

Proof. Suppose that $f_{T}$ is the integral mean of $f$ over $\Omega \cap \sigma_{T}$ and calculate

$$
\left\|h_{T} f\right\|_{L^{2}\left(\Omega \cap \sigma_{T}\right)}^{2}-\left\|h_{T}\left(f-f_{T}\right)\right\|_{L^{2}\left(\Omega \cap \sigma_{T}\right)}^{2}=\left\|h_{T} f_{T}\right\|_{L^{2}\left(\Omega \cap \sigma_{T}\right)}^{2} .
$$

Assumption 6.3 implies that $\left|\sigma_{T}\right| \lesssim h_{T}^{2}$ and that (7.6) is bounded by

$$
\begin{aligned}
h_{T}^{2}\left\|b_{T}^{1 / 2} f_{T}\right\|_{L^{2}\left(\Omega \cap \sigma_{T}\right)}^{2} \lesssim h_{T}^{2}\left|f_{T}\right| \int_{\Omega \cap \sigma_{T}} b_{T} \mid & f_{T}-f \mid d x \\
& +h_{T}^{2}\left|f_{T} \int_{\Omega \cap \sigma_{T}} b_{T} f d x\right| .
\end{aligned}
$$


This and Cauchy's and Young's inequalities lead to

$$
\begin{aligned}
\left\|h_{T} f\right\|_{L^{2}(\Omega \cap T)}^{2} & \leq\left\|h_{\mathcal{T}} f\right\|_{L^{2}\left(\Omega \cap \sigma_{T}\right)}^{2} \\
& \lesssim\left\|h_{T}\left(f-f_{T}\right)\right\|_{L^{2}\left(\Omega \cap \sigma_{T}\right)}^{2}+h_{T}^{2}\left(\int_{\Omega \cap \sigma_{T}} b_{T} f d x\right)^{2} .
\end{aligned}
$$

We focus on the estimate of $\int_{\Omega \cap \sigma_{T}} b_{T} f d x$. By choosing $z^{\star}=b_{T}$ in (5.9), we obtain

$$
\begin{aligned}
& \int_{\Omega \cap \sigma_{T}} f b_{T} d x=\int_{\Omega \cap \sigma_{T}} \nabla e \cdot \nabla b_{T} d x \\
& \quad-\sum_{\omega \in \mathcal{C}}\left(\int_{\partial \omega}\left(b_{T}-c_{\omega}\right) \frac{\partial U^{\star}}{\partial n_{\omega}} d s+\int_{\omega \cap(\cup \mathcal{E})}\left(b_{T}-c_{\omega}\right)\left[\frac{\partial U^{\star}}{\partial n_{\mathcal{E}}}\right] d s\right) .
\end{aligned}
$$

Next, we choose the constants $c_{\omega}$ in (7.9). For all holes $\omega \in \mathcal{C}$ which are compactly included in $\sigma_{T}$, we choose $c_{\omega}:=\int_{\partial \omega} b_{T} d s /|\partial \omega|$ and observe

$$
\left\|b_{T}-c_{\omega}\right\|_{L^{2}(\partial \omega)} \lesssim|\partial \omega|^{1 / 2} h_{\omega} / h_{T}
$$

For all remaining holes, we choose $c_{\omega}=0$ and, since $b_{T}$ vanish at some point of $\partial \omega$ in these cases, we get the estimate

$$
\left\|b_{T}-c_{\omega}\right\|_{L^{2}(\partial \omega)}=\left\|b_{T}\right\|_{L^{2}(\partial \omega)} \lesssim|\partial \omega|^{1 / 2} h_{\omega} / h_{T} .
$$

Thus, the last sum in (7.9) vanishes while the second last sum can be estimated form above by

$$
\begin{aligned}
& \sum_{\omega \in \mathcal{C}} \int_{\partial \omega}\left|\left(b_{T}-c_{\omega}\right) \frac{\partial U^{\star}}{\partial n_{\omega}}\right| d s \leq \sum_{\omega \in \mathcal{C}}\left\|b_{T}-c_{\omega}\right\|_{L^{2}(\partial \omega)}\left\|\frac{\partial U^{\star}}{\partial n_{\omega}}\right\|_{L^{2}(\partial \omega)} \\
& \lesssim \sum_{\omega \in \mathcal{C}_{\sigma_{E}}}|\partial \omega|^{1 / 2} \frac{h_{\omega}}{h_{T}}\left\|\frac{\partial U^{\star}}{\partial n_{\omega}}\right\|_{L^{2}(\partial \omega)} \lesssim\left(\sum_{\omega \in \mathcal{C}_{\sigma_{E}}}\left\|h_{\omega}^{1 / 2} \frac{\partial U^{\star}}{\partial n_{\omega}}\right\|_{L^{2}(\partial \omega)}^{2}\right)^{1 / 2}
\end{aligned}
$$

The combination of those estimates concludes the proof.

Lemma 7.5. Under Assumptions 6.1, 6.4, 6.5, 6.7, 6.8 we have, for any $f_{\omega} \in \mathbb{R}, f_{L}^{\star}:=\Delta \mathfrak{E}_{L} u$, and $V_{\omega}^{\star \star}$ as in (6.1),

$$
h_{\omega}^{1 / 2}\left\|\partial U^{\star} / \partial n\right\|_{L^{2}(\partial \omega)} \lesssim\left|\mathfrak{E}_{L} e\right|_{H^{1}\left(V_{\omega}^{\star \star}\right)}+h_{\omega}\left\|f_{L}^{\star}-f_{\omega}\right\|_{L^{2}\left(V_{\omega}^{\star \star}\right)} .
$$

Proof. Abbreviate $q_{T}:=\left.\nabla U^{\star}\right|_{T} \in \mathbb{R}^{2}$ for $T \in \mathcal{T}$ and obtain

$$
\int_{\partial \omega}\left|\partial U^{\star} / \partial n\right|^{2} d s=\sum_{T \in \mathcal{T}_{\omega}} \int_{T \cap \partial \omega}\left|q_{T} \cdot n\right|^{2} d s \lesssim \max _{T \in \mathcal{T}_{\omega}} \int_{T \cap \partial \omega}\left|q_{T} \cdot n\right|^{2} d s
$$


from card $\mathcal{T}_{\omega} \lesssim 1$. Let the maximum on the right-hand side of (7.10) be attained for $K \in \mathcal{T}$. Hence,

$$
\int_{\partial \omega}\left|\partial U^{\star} / \partial n\right|^{2} d s \lesssim \int_{\partial \omega}\left|q_{K} \cdot n\right|^{2} d s
$$

Let $\alpha$ denote the polar angle of $q_{K} \in \mathbb{R}^{2}$ and define $\lambda=\lambda_{\alpha}$ as in Assumption 6.6. Combining $\partial u / \partial n=0$ on $\gamma$, the last condition in Assumption 6.6, and the divergence theorem we derive, for any $f_{\omega} \in \mathbb{R}$,

$$
\begin{aligned}
& \left\|q_{K} \cdot n\right\|_{L^{2}(\partial \omega)}^{2} \lesssim \int_{\partial \omega}\left(q_{K} \cdot N\right)\left(q_{K} \cdot n\right) d s \\
& =\int_{\partial \omega}\left(q_{K} \cdot N\right)\left(\left(q_{K}-\nabla u\right) \cdot n\right) d s \\
& =\int_{V_{\omega}^{i n t}}\left\{\left(\nabla\left(q_{K} \cdot N\right)\right)\left(q_{K}-\nabla u\right)+\left(q_{K} \cdot N\right)\left(f-f_{\omega}\right)\right\} d x .
\end{aligned}
$$

With Assumption 6.7, we infer

$$
\begin{aligned}
\left\|q_{K} \cdot n\right\|_{L^{2}(\partial \omega)}^{2} \lesssim & \left\|\left(q_{K} \cdot n\right)\right\|_{L^{2}(\partial \omega)} h_{\omega}^{-1 / 2}\left\|q_{K}-\nabla u\right\|_{L^{2}\left(V_{\omega}^{i n t}\right)} \\
& +\left\|\left(q_{K} \cdot n\right)\right\|_{L^{2}(\partial \omega)} h_{\omega}^{1 / 2}\left\|f-f_{\omega}\right\|_{L^{2}\left(V_{\omega}^{i n t}\right)} .
\end{aligned}
$$

The combination of (7.12) with (7.10) yields

$$
h_{\omega}^{1 / 2}\left\|\partial U^{\star} / \partial n\right\|_{L^{2}(\partial \omega)} \lesssim\left\|q_{K}-\nabla u\right\|_{L^{2}\left(V_{\omega}^{i n t}\right)}+h_{\omega}\left\|f-f_{\omega}\right\|_{L^{2}\left(V_{\omega}^{i n t}\right)} .
$$

It remains to consider the first term on the right-hand side. Since

$$
\left\|q_{K}-\nabla u\right\|_{L^{2}\left(V_{\omega}^{i n t}\right)} \leq\|\nabla e\|_{L^{2}\left(V_{\omega}^{i n t} \cap K\right)}+\left\|q_{K}-\nabla u\right\|_{L^{2}\left(V_{\omega}^{i n t} \backslash K\right)}
$$

it is sufficient to investigate the last term in (7.13). Take the sequence of edge-connected triangles $\left(K_{j}\right)_{j=0}^{J}$ as in Assumption 6.8 and recall the definition of the balls $D_{j}$ with radii $r_{j}$ therein. Put $q_{j}:=q_{K_{j}}$ and notice that continuity of $U^{\star}$ along $E_{j}$ implies that $q_{j}-q_{j-1}$ is parallel to $n_{E_{j}}$. Then,

$$
\left|q_{j}-q_{j-1}\right|=\left|\left[\partial U^{\star} / \partial n_{E_{j}}\right]\right| \quad \text { for } j=1,2, \ldots, J .
$$

Define a bubble function $b_{j}$ supported in $D_{j}$ with

$$
\begin{aligned}
\left\|b_{j}\right\|_{L^{\infty}\left(D_{j}\right)}+r_{j}\left|b_{j}\right|_{W^{1, \infty}\left(D_{j}\right)} & \lesssim 1, \\
\int_{D_{j} \cap E_{j}} b_{j} d s & \approx r_{j}, \quad \text { and } \quad \int_{D_{j}} b_{j}=0 .
\end{aligned}
$$

Put $u^{\star}:=\mathfrak{E}_{L} u, f_{L}^{\star}:=-\Delta u^{\star}$, and $e_{L}^{\star}:=u^{\star}-U^{\star}$. Remark 6.9 implies $e_{L}^{\star}:=\mathfrak{E}_{L} e$ on $D_{j}$. An integration by parts shows, as in the proof of 
Lemma 7.2 , by using (6.2)

$$
\begin{aligned}
h_{\omega}\left|\left[\partial U^{\star} / \partial n_{E_{j}}\right]\right| & \approx\left|\int_{D_{j} \cap E_{j}} b_{j}\left[\partial U^{\star} / \partial n_{E_{j}}\right] d s\right| \\
& =\left|\int_{D_{j}}\left(\nabla b_{j} \cdot \nabla e^{\star}-f_{L}^{\star} b_{j}\right) d x\right| \\
& \lesssim\left|e_{L}^{\star}\right|_{H^{1}\left(D_{j}\right)}+h_{\omega}\left\|f_{L}^{\star}-f_{\omega}\right\|_{L^{2}\left(D_{j}\right)} .
\end{aligned}
$$

The combination of (7.14)-(7.15) results in

$$
\begin{aligned}
\left\|q_{j}-q_{j-1}\right\|_{L^{2}\left(V_{\omega}^{i n t}\right)} & \lesssim h_{\omega}\left|\left[\partial U^{\star} / \partial n_{E_{j}}\right]\right| \\
& \lesssim\left|e_{L}^{\star}\right|_{H^{1}\left(D_{j}\right)}+h_{\omega}\left\|f_{L}^{\star}-f_{\omega}\right\|_{L^{2}\left(D_{j}\right)} .
\end{aligned}
$$

Owing to $J \lesssim 1$, triangle inequalities lead to

$$
\left\|q_{K}-\nabla u\right\|_{L^{2}\left(V_{\omega}^{i n t} \cap T\right)} \leq\left|e_{L}^{\star}\right|_{H^{1}\left(V_{\omega}^{\star \star}\right)}+\sum_{j=1}^{J}\left\|q_{j}-q_{j-1}\right\|_{L^{2}\left(V_{\omega}^{i n t}\right)} .
$$

Utilizing (7.16)-(7.17) and summing the result for all $T \in \mathcal{T} \backslash\{K\}$ with $T \cap V_{\omega}^{\text {int }} \neq \emptyset$ we conclude

$$
\left\|q_{K}-\nabla u\right\|_{L^{2}\left(V_{\omega}^{i n t} \backslash K\right)} \lesssim\left|e_{L}^{\star}\right|_{H^{1}\left(V_{\omega}^{\star \star}\right)}+h_{\omega}\left\|f_{L}^{\star}-f_{\omega}\right\|_{L^{2}\left(V_{\omega}^{\star \star}\right)} .
$$

The combination with (7.13) concludes the proof.

Lemma 7.6. Under Assumptions 6.1, 6.4, 6.5, 6.7, 6.8 we have, for any $f_{\omega} \in \mathbb{R}, f_{L}^{\star}:=\Delta \mathfrak{E}_{L} u$, and $V_{\omega}^{\star}$ as in (6.1),

$$
h_{\omega}^{1 / 2}\left\|\left[\partial U^{\star} / \partial n_{\mathcal{E}}\right]\right\|_{L^{2}(\omega \cap(\cup \mathcal{E}))} \leq\left|e^{\star}\right|_{H^{1}\left(V_{\omega}^{\star \star}\right)}+h_{\omega}\left\|f_{L}^{\star}-f_{\omega}\right\|_{L^{2}\left(V_{\omega}^{\star \star}\right)} .
$$

Proof. We adopt the notations of the previous proof. Consider any $E \in \mathcal{E}$ satisfying $|E \cap \omega|>0$. The estimate

$$
h_{\omega}\left|\left[\partial U^{\star} / \partial n_{E}\right]\right| \lesssim\left|e^{\star}\right|_{H^{1}\left(D_{j}\right)}+h_{\omega}\left\|f_{L}^{\star}-f_{\omega}\right\|_{L^{2}\left(D_{j}\right)}
$$

is derived as (7.15). By employing $\sqrt{h_{\omega}|\omega \cap E|} / h_{\omega} \lesssim 1$ we obtain

$$
h_{\omega}^{1 / 2}\left\|\left[\partial U^{\star} / \partial n_{E}\right]\right\|_{L^{2}(\omega \cap E)} \lesssim\left|e^{\star}\right|_{H^{1}\left(V_{\omega}^{\star \star}\right)}+h_{\omega}\left\|f_{L}^{\star}-f_{\omega}\right\|_{L^{2}\left(V_{\omega}^{\star}\right)} .
$$

Since, for any hole $\omega \in \mathcal{E}$, the number of edges $E$ with $|E \cap \omega|>0$ is bounded by a moderate constant (cf. (5.1)) a summation of (7.19) over all $E \in \mathcal{E}$ leads to (7.18). 


\section{REFERENCES}

[BS] S.C. Brenner, L.R. Scott: The Mathematical Theory of Finite Element Methods. Texts Appl. Math. 15, Springer, New-York, 1994.

[CB] C. Carstensen, S. Bartels: Each averaging technique yields reliable a posteriori error control in FEM on unstructured grids. Part I: Low order conforming, nonconforming and Mixed FEM. Math. Comp. (accepted (2001)). Preprint available at http://www.numerik.uni-kiel.de/reports/1999/.

[CF] C. Carstensen, S.A. Funken: Constants in Clément-interpolation error and residual-based a posteriori error estimates in Finite Element Methods. EastWest-Journal of Numerical Analysis 8 (2000) 153-175.

[Ci] P.G. Ciarlet: The finite element method for elliptic problems. NorthHolland, Amsterdam 1978.

[Cl] P. Clément: Approximation by finite element functions using local regularization. RAIRO Sér. Rouge Anal. Numér. R-2 (1975) 77-84.

[DR] W. Dörfler, M. Rumpf: An adaptive strategy for elliptic problems including a posteriori controlled boundary approximation. Math. Comp. 67 (1998), 1361-1382.

[EEHJ] K. Eriksson, D. Estep, P. Hansbo, C. Johnson: Computational Differential Equations. Cambridge, Univ. Press, 1996.

[G] P. Grisvard: Elliptic problems in nonsmooth domains. Pitman, 1985

[HS1] W. Hackbusch, S.A. Sauter: Composite finite elements for the approximation of PDEs on domains with complicated micro-structures. Numer. Math. 75, 1997, 447-472.

[HS2] W. Hackbusch, S.A. Sauter: Composite finite elements for problems containing small geometric details. Part II: Implementation and numerical results. Comput. Visual. Sci. 1, 1997, 15-25.

[N] J. Nečas: Let methodes directes en theorie des equations elliptiques. Academia, Prague, 1967.

[OR] M. Ohlberger, M. Rumpf: Hierarchical and adaptive visualization on nested grids. Computing 59 (1997), 365-385.

[OSY] O.A. Oleinik, A.S. Shamaev and G.A. Yosifian: Mathematical problems in elasticity and homogenization. North-Holland, Amsterdam, 1992.

[PW] L.E. Payne, H.F. Weinberger: An optimal Poincaré-inequality for convex domains. Archiv Rat. Mech. Anal. 5 (1960) 286-292

[SW] S.A. Sauter, R. Warnke: Extension operators and approximation on domains containing small geometric details. East-West J. Numer. Math. 7 (1999) 61-78.

[S] E.M. Stein: Singular integrals and differentiability properties of functions. Princeton, Univ. Press, N.J. 1970.

[V] R. Verfürth: A review of a posteriori error estimation and adaptive mesh-refinement techniques. Teubner Skripten zur Numerik. B.G. WileyTeubner, Stuttgart, 1996. 
Mathematisches Seminar, Christian-Albrechts-Universität Zu Kiel, LudewigMeyn-Str. 4, D-24098 Kiel, Germany

E-mail address: cc@numerik.uni-kiel.de

Institut FÜr MAThematiK, Universität ZÜRICh, Winterthurerstr. 190, CH-8057 Zürich, Switzerland.

E-mail address: stas@amath.unizh.ch 